HELENA SIWICKA-TARWIDOWA.

Inst. de Bot. Générale de l'Univ. de Varsovie.

\title{
SUR L'ÉVOLUTION DU CHONDRIOME PENDANT LE DÉVELOPPEMENT DU SAC EMBRYONNAIRE DE L'ORCHIS LATIFOLIUS L.
}

\section{Matériel et technique.}

Ayant à étudier l'évolution du chondriome pendant le développement du sac embryonnaire, j'ai pris pour objet de mes observations l'Orchis latifolius L. et cela pour les raisons suivantes:

La cellule primordiale du sac embryonnaire jusqu'aux premiers stades de la prophase de la mitose réductrice (le stade plus ou moins de synizesis) n'est enveloppée que d'une couche de cellules épidermiques, car les téguments à cette époque commencent seulement à apparaître; elle constitue un excellent matériel d'étude vitale. Dans les stades postérieurs, gràce aux intéguments qui sont extrêment minces et qui n'ont a peine que quelques couches de cellules, les fixateurs peuvent y pénétrer rapidement et l'on obtient alors des préparations exceptionnellement belles.

L'orchidée est en outre une plante qui n'a pas d'amidon dans ses ovules mais seulement des graisses, ce qui m'a permis de faire une analyse exacte du rôle que joue le chondriome au cours de la production des dits composés chimiques. 
Conformement à ce que dit Vermoesen (31), le schéma du développement du sac embryonnaire de l'Orchis latifolius L. est le suivant: La cellule primordiale se différencie directement au dépens de la cellule terminale sous-épidermique de la rangée cellulaire interne des filaments ovulaires; elle ne forme pas de cellules de la calotte. Par deux cinèses de maturation, elle forme une tétrade incomplète, c'est à dire trois cellules de mégaspores (la cellule extérieure de la dyade ne se divise pas). La cellule intérieure des mégaspores se développe en un sac embryonnaire normal, contenant huit noyaux. Les deux autres cellules de cette tétrade dégénèrent.

J'ai fixé l'ovaire de l'Orchis latifolius L. (coupé en deux parties) d'après la méthode de Regaud, de Benda et d'après celle de Lewitsky. La méthode de Regaud n'a donné que des résultats médiocres, tandis que celle de Benda m'en a apporté de tout à fait satisfaisants.

Il convient de faire remarquer que le meilleur fixateur pour l'objet de mes observations a été celui de Lewitsky (maintenir deux jours et demi dans la solution de formol à 10 pour 100 et dans l'acide chromique à 1 pour 100 dans la proportion de 9:1; garder ensuite cinq jours dans la solution suivante: cinq parties d'acide chromique à 1 pour 100, deux parties d'acide osmique à 1 pour 100 et treize parties d'eau).

Je colorais les coupes microtomiques, épaisses de 7 à 10 microns, à l'bématoxyline ferrique de Heidenhain, ainsi qu'à la fuchsine et à la safranine. Dans l'emploi de la fuchsine acide j'appliquais la méthode suivante de la double coloration: 1) la fuchsine acide chauffée jusqu'à la première vapeur et l'aurantia, 2) la fuchsine acide à chaud et l'acide picrique. En appliquant ces deux méthodes, je n'ai pas réussi à obtenir une coloration satisfaisante.

En réduisant au minimum la durée de séjour des préparations (colorées au préalable à la fuchsine) dans la solution d'aurantia et dans de l'acide picrique (par plongement et prompt déplacement de la lame dans de l'eau) et en appliquant différentes concentrations, j'ai obtenu dans la plupart de cas des préparations sur lesquelles le chondriome était complètement décoloré, ou bien coloré à la fuchsine acide, 
mais le plasma ne se colorait ni à l'aurantia ni à l'acide picrique.

Pour obtenir une double coloration à la fuchsine acide et au vert de méthyle, j'agissais de la façon suivante: je réchauffais la préparation dans de la fuchsine acide jusqu'à la première vapeur, je la gardais 5 minutes dans cette température, ensuite je la rincais quelques minutes dans de l'eau distillée et enfin je la colorais au vert de méthyle à 1 pour 100, par prompte immersion.

Motte (25) gardait ses préparations de 2 à 3 minutes dans du vert de méthyle. Sur les miennes le chondriome, après une telle durée, était complètement décoloré.

L'application de cette méthode m'a donné de très bons résultats, car j'ai réussi à obtenir des préparations sur lesquelles le chondriome est coloré en rouge à la fuchsine acide et se distingue nettement sur le fond verdàtre du plasma; de plus, le noyau est coloré en vert et le nucléole en rouge. Lors de la coloration à la safranine, le plasma a été légerement teinté dans la solution de vert-lumière à 25 pour 100 .

Ces expériences m'ont conduit à constater, que les résultats obtenus par la fixation et la coloration des préparations microscopiques étaient très différents selon le cas. Probablement cette diversité est dùe à la sensibilité extraordinaire des préparations aux moindres détails de leur exécution. Il faut en outre supposer que l'état physico-chimique de la cellule, au moment de la fixation et de la coloration, joue aussi un rôle très important.

J'ai pu également observer le fait, que très souvent les résultats dépendaient de l'emploi du matériel. J'ai été donc forcée de modifier, selon le besoin, toutes les méthodes connues, car les méthodes appliquées à d'autres objets se montraient défectueuses pour mon matériel.

Les images obtenues par la coloration à l'hématoxyline ferrique de Heidenhain et à la fuchsine acide, sont en principe presque identiques à celles que l'on obtient, si l'on applique des colorations vitales. Seules les préparations colorées à l'hématoxyline diffèrent des autres en ce que les structures observées sont légèrement gonflées.

Aussi, en m’appuyant sur cette identité des images obtenues, j'ai exécuté les figures (le matériel fixé) uniquement d'après les préparations colorées à l'hématoxyline, en tenant 
compte de ce qu'elles sont plus claires et plus contrastées. En égard à la très petite dimension des matériaux étudiés, j'ai dû me servir de très forts appareils optiques. Les préparations colorées à la fuchsine ou à la safranine donnaient sous les forts grossissements des images peu contrastées et confuses.

Dans les figures tout est représenté d'une manière schématique, sauf le chondriome et la graisse, qui est en étroit rapport avec lui.

Le cytoplasme, fixé d'après la méthode de Levitsky, très fortement différencié après l'hématoxyline ferrique et dessiné sous un éclairage très fort, n'offre aucune structure; il est clair et homogène.

En ce qui concerne le noyau, il se colore à certains stades tout à fait bien, tandis qu'à d'autres stades où la forte différenciation est favorable pour le chondriome, le noyau est incoloré.

Je partageais les coupes microtomiques, obtenues de chaque ovaire coupé, en deux parties. Je plongeais l'une de ces parties pour quelques heures dans de l'eau oxygénée, afin d'en décolorer la graisse (le fixateur de Lewitsky contient de l'acide osmique, qui est réduit par la graisse) et je la colorais selon les méthodes mentionnées plus haut. La seconde partie de l'ovaire fut utilisée pour l'étude des graisses sans être préalablement oxygénée.

\section{Observations sur le matériel fixé.}

J'ai commencé mes observations à partir de la période où la cellule primordiale du sac embryonnaire ne diffère pas encore, au point de vue de la dimension, des cellules de l'ovule qui l'entourent.

Les chondriocontes sont en forme de bàtonnets et de filaments longs ou courts, par endroits non uniformement épais (fig. 1 Pl. VII). A côté des chondriocontes apparaissent en petit nombre des grains minuscules qui, comme le prouve l'emploi de la vis micrométrique, ne constituent pas la coupe transversale des chondriocontes. On peut supposer, que ce sont des bouts de filament coupés à l'aide du microtome et cela est d'autant plus possible qu'ils apparaissent à ces sta- 
des très rarement. Il y a aussi de nombreuses cellules dans lesquelles on ne voit ipoint de mitochondries granuleuses. Cela est remarquable dans la figure 2, Pl. VII qui représente le chondriome au stade correspondant aux cellules épidermiques de l'ovule.

Dans la seconde période d'accroissement de la cellule primordiale et au cours des premiers stades de la prophase réductrice (leptotène, zygotène) le chondriome subit diverses transformations morphologiques. Il en résulte, que les chondriocontes, jusqu'ici en forme de filaments et de bâtonnets, prennent la forme de gros et de petis grains (fig. 7 Pl. VII) qui, comme les chondriocontes aux stades les plus précoces, se colorent intensivement à l'hématoxyline.

Entre ces deux états morphologiques du chondrioconte, il y a toute une série de formes transitoires. (fig. 3, 4, 5, 6 PI. VII). On voit donc les courtes plaquettes et les batonnets (fig. 3 et $5 \mathrm{Pl}$. VII) qui naissent probablement par la fragmentation de longs filaments, ou par leur contraction.

Ces plaquettes et ces bàtonnets ont la forme de virgules, lorsqu'ils ne sont renflés que sur l'une de leurs extrémités; s'ils sont renflés à deux extrémités, ils prennent alors la forme de biscuits. Les renflements plus forts donnent des éléments en forme de poires. (Pl. VII fig. 4, 5 et 6 ).

Enfin, un filament peut subir des renflements dans plusieurs endroits. Dans la plupart des cas les parties de ce filament qui se trouvent entre ces renflements deviennent de plus en plus minces jusqu'à ce qu'elles soient interrompues en fournissant toute une série de grains placés l'un à côté de l'autre.

Je dois encore faire remarquer qu'à côté d'une quantité prépondérente de gros grains on voit aussi de courtes plaquettes et de tout petits grains (Pl. VII fig. 7) qui deviennent maintenant beaucoup plus nombreux qu'ils n'étaient aux stades précédents.

Il en résulte que le chondriome de la cellule primordiale de l'Orchis latifolius L. subit au premier stade d'accroissement toute une série de transformations qui se poursuivent dans un ordre déterminé (O rmann (28), Guillie rmond $(5,7$, 14, 16, 17), Lewitsky (1911), Wagner $(32,33)$, Py $(29,30)$ et d'autres).

A la fin du zygotène ou au commencement de la syni- 
zèse, des gros grains, se colorant jusqu'alors intensivement en noir à l'hématoxyline ferrique, changent leur état chimique (ou physique) en sorte qu'ils changent leur coloration et de noirs ils deviennent gris. En même temps les dimensions des grains augmentent (fig. 8, 9, 10 Pl. VII). Les éléments en forme de têtards et de poires subissent ces mêmes métamorphoses (fig. 9, $10 \mathrm{Pl}$. VII).

Aux premiers moments de cette transformation, à côté des éléments colorés en gris, il y a encore des grains et des éléments en forme de têtards et de virgules plus fortement teintés qui subissent également les mêmes transformations progressives.

Ils deviennent de moins en moins nombreux (voir fig. 8, 9 , $10 \mathrm{Pl}$. VII) jusqu'à ce qu'enfin il n'en reste que de tout petites plaquettes et des petits grains peu nombreux, colorés fortement en noir.

Tous les stades étudiés jusqu'ici, observés en même temps sur les préparations correspondantes non oxygénées, ne démontrent pas la présence de gouttes de graisse.

La période de la synizèse est de très longue durée. Au cours de cette période les grains pleins, colorés en gris, donnent naissance aux petites vésicules, remplies d'une substance non colorée, ce qui donne, sur les coupes, l'impression de petits anneaux colorés en gris (fig. 11, 12, 13 Pl. VII) Ces anneaux sont d'abord petits et ils sont entourés par une paroi épaisse. Peu à peu la goutte, qui se trouve à l'intérieur, prend des dimensions plus grandes; les parois de la vésicule deviennent de plus en plus minces et par suite on obtient sur les préparations des anneaux plus grands a contours plus délicats (fig. 12, $13 \mathrm{Pl}$. VII). On peut observer le même fait dans tous les autres éléments du chondriome, que ce soient les éléments en forme de têtards, de poires ou de grains liés. C'est pour cela qu'on a sur les coupes optiques le chondriome en forme de raquettes de tennis (fig. 12, 13 Pl. VII) et d'anneaux liés entre eux (fig. 12 Pl. VII).

A côté de ces éléments il y a dans le cytoplasme, au cours de ce stade comme au cours des stades précédents, une quantité innombrable de plaquettes et de petits grains se colorant fortement à l'hématoxyline ferrique.

En même temps, les préparations non oxygénées des mêmes stades montrent l'apparition progressive des gouttes 
minuscules de graisse colorées à l'osmium, petites et peu nombreuses tout d'abord (fig. 14, 15 Pl. VII), mais qui deviennent de plus en plus grandes et plus nombreuses ${ }^{1}$ ) (fig. 16, 17 Pl. VII).

Souvent on rencontre aussi des gouttes rangées deux a deux ou en forme de chaînettes; enfin il y en a celles, qui par leur forme rappellent le chondriome en forme de poires et de raquettes de tennis.

Dans les parties vertes de l'ovaire, la graisse apparaît àu fond de grands chloroplastes. En mème temps les plastes subissent les mêmes transformations que le chondriome dans la cellule primordiale, a savoir: les grands chloroplastes, qui sont en forme de fuseau et colorés en noir à l'hématoxyline, deviennent sphériques, augmentent de dimension et en même temps ils se colorent peu à peu en gris. C'est le moment qui précède immédiatement l'apparition d'une grosse goutte de graisse et où le plaste donne sur la coupe un grand anneau.

Puisqu'il n'y a pas ici une telle périodicité dans la production en masse de la graisse, comme au cours du développement du sac embryonnaire, on peut trouver en même temps tous les stades de la transformation des plastes. Quel sort subissent les chloroplastes après la dissolution de la graisse qui s'y est produite, je ne puis pour le moment affirmer rien de définitif.

Pour obtenir des préparations plus parfaites j'ai essayé de colorer le contenu des vésicules chondriomales à l'aide du Sudan III sur les préparations colorées à l'hématoxyline.

Cependant le Sudan III, qui donnait de très bons résultats sur le vivant, n'a pas déterminé de coloration nette, probablement à cause des transformations, qui ont eu lieu sous l'influence du fixateur dans la nature chimique de la graisse.

Comme la méthode précédente ne m'a pas donnée de résultats positifs, j’ai appliqué un tout autre procédé: sur les préparations contenants de petites gouttes de graisse, colo-

1) Le chondriome, comme l'a démontré F a u ré-F re mi e t dans les cellules animales et Guilliermond dans les celles des plantes, ne reduit pas l'acide osmique, exepté le cas où on le traite ensuite par une solution de pyrogallol ou lorsqu'on applique la méthode d'imprégnation osmique (laisser séjourner deux semaines à la température de $40^{\circ}$ dans l'acide osmique à 2 pour 100). 
rées en brun à l'osmium, j'ai me suis efforcée de mettre en evidence les vésicules chondriomales, qui entouraient ces gouttes, en employant de la fuchsine acide (La teinte grise, obtenue à l'aide de l'hématoxyline, ne se laisse pas distinguer de la graisse colorée en brun à l'osmium). Mais le chondriome perd dans ce stade non seulement l'affinité pour l'hématoxyline, mais il se colore aussi faiblement à la fuchsine acide, de manière que les préparations obtenues ne sont pas tout à fait claires.

Avec le temps les parois des vésicules chondriomales deviennent de plus en plus minces jusqu'à ce qu'elles disparaissent complètement. En même temps se dissolvent les gouttes de graisse, car sur les préparations osmiques rien ne se colore plus. Au commencement du pachytène, le chondriome de la cellule primordiale est représenté seulement par des grains minuscules des mitochondries, colorés en noir (fig. 18, $19 \mathrm{Pl}$. VIII). Ce n'est que très rarement qu'on peut observer pendant ce stade des anneaux particuliers, très peu nombreux (fig. $19 \mathrm{Pl}$. VIII).

Le trait caractéristique de ces grains minuscules est qu'ils sont le plus souvent reliés par paires au moyen des filaments très minces colorés en gris (très rarement ces filaments sont colorés en noir).

La longueur des filaments, qui lient ces grains, est très différente; ils sont très courts et les grains se trouvent l'un tout près de l'autre, ou, au contraire, ils sont très longs et alors ils forment entre les grains une ligne droite, ou courbée en forme de faucile (Pl. VIII fig. 18, 19, 20). A côté de tels éléments, il y a encore des corpuscules en forme de bisquits, c'est à dire de petits grains colés l'un à l'autre. Ceux-ci font l'impression de stades de la division du chondriome (fig. 18, 19, 20 Pl. VIII). Le fait est que le chondriome s'y multiplie, car le nombre de grains minuscules augmente visiblement, ce qui s'observe parfaitement en comparant les stades successifs du pachytène (PI. VIIIf ig. 18, 19), du strepsitène (fig. 20 Pl. VIII), du diplotène (fig. 21 Pl. VIII) et de la métaphase de la mitose hétérotypique (fig. 22 Pl. VIII). Ce problème sera discuté dans les conclusions. J'ai pu encore constater, que pendant la division du noyau, le comportement du chondriome est tout à fait passif.

Aussi bien dans les derniers moments de la prophase 
de la mitose hétérotypique (fig. 18, 19, 20, 21 PI. VIII) que dans la métaphase elle-même (fig. 22 Pl. VIII) les éléments en forme de biscuits, les grains liés entre eux et les grains libres sont tout à fait uniformement répartis sur tout le cytoplasme.

Pendant la période de la métaphase et de l'anaphase la région du fuseau reste libre de chondriome.

Wagner (32) a constaté la même chose pour les grains de pollen des Monocotylédones, où le chondriome, pendant le premier et le deuxième stade de la division de la cellule-mère, est uniformement réparti sur tout le cytoplasme de la cellule; seule la région du fuseau en est libre.

En conséquence, le cytoplasme de deux cellules de la dyade contient, par rapport à sa masse, une quantité égale de chondriome. Mais puisque le noyau de la cellule primordiale, déjà au commencement de la prophase de la mitose réductrice, passe de sa position centrale (fig. $3 \mathrm{Pl}$. VII) vers la paroi extérieure (fig. $11 \mathrm{Pl}$. VII), par suite de quoi dans la métaphase la plaque chromosomique occupe plus ou moins deux tiers de la longueur de la paroi avoisinante des cellules du funicule et un tiers de la paroi opposée, la cellule extérieure de la dyade est plus petite et par conséquent elle contient une quantité plus petite de chondriome (fig. 23, 24 Pl. VIII).

Dans les cellules de la dyade, immédiatement après la mitose hétérotypique, le chondriome, qui y est contenu, garde le caractère qu'il avait aux stades précédents, mais au fur et à mesure que les cellules commencent à se préparer à la mitose homéotypique (cela a lieu probablement immédiatement après la mitose hétérotypique, car je n'ai pas réussi à saisir le noyau à l'état de repos) commencent à apparaître dans les deux cellules, à côté du chondriome, décrit plus haut, de courts chondriocontes recourbés et souvent même un peu renflés sur l'une de leurs extrémités ou sur les deux (fig. 24 Pl. VIII).

Au cours de la métaphase de la mitose homéotypique on voit dans les deux cellules de la dyade (fig. 25 Pl. VIII) une forte augmentation du nombre des éléments filamenteux. Ces filaments sont fortement renflés sur leur extrémités, ou en quelques autres endroits. En outre, il y a de courtes plaquettes non uniformement' renflées, de gros grains, de filaments longs et minces et enfin des grains minuscules. A partir de ce moment, le chondriome subit strictement les mêmes trans- 
formations, que dans la cellule primordiale avant la première phase de la formation de la graisse.

$\mathrm{Au}$ cours de la métaphase de la mitose homéotypique, le chondriome, comme cela avait lieu pendant la mitose hétérotypique, est en général uniformement reparti dans le cytoplasme. On remarque assez souvent que des éléments de plus grandes dimensions se groupent plutôt dans la cellule inférieure de la dyade, au pôle contigu au funicule.

Au cours de la télophase (fig. $26 \mathrm{Pl}$. VIII) on voit des éléments de plus en plus nombreux en forme de spermatozoïdes têtards, ainsi que des corpuscules granuleux.

La cellule extérieure de la dyade s'attarde dans sa division (fig. $26 \mathrm{Pl}$. VIII); elle reste toujours au stade de la métaphase et n'atteint, que très rarement, celui de l'anaphase (fig. 28 Pl. VIII).

Le chondriome des tétraspores garde à peu près le mêne caractère qu'il avait au cours des stades précédents. Dans la figure 27 (PI. VIII) on voit nettement la tétraspore inférieure, c'est à dire la cellule-mère du sac embryonnaire, et la tétraspore centrale. La tétraspore extérieure avec la plaque métaphasique qui dégénère est, au contraire, masquée fortement par l'hématoxyline. Le chondriome de la tétraspore extérieure dégénérescente (fig. $28 \mathrm{Pl}$. VIII) n'est composé que de granules assez gros. Les plus petits de ces granules sont encore beaıcoup plus gros que les granules de la cellule-mère, dans laquelle, à côté de gros grains, apparaissent en même temps ces grains minuscules et de petites plaquettes (fig. 28, 29 Pl. VIII).

Pendant la période d'accroissement de la cellule-mère du sac embryonnaire, (le noyau de cette cellule est encore à l'état de repos); de gros grains, des éléments en forme de têtards et ceux en forme de poires etc. augmentent leurs dimensions plus fortement, que ceux de la cellule primordiale, et se colorent en gris à l'hématoxyline (fig. 30 Pl. VIII).

Je dois encore attirer l'attention sur le fait qu'à ce stade comme au stade suivant il est extrèmement difficile de rendre visible le chondriome. Les préparations doivent être très fortement différenciées (aprés l'hémotoxyline) en sorte que le noyau, et même le nucléole sont sur certaines coupes tout à fait décolorés (fig. 30,32 Pl. VIII). J'ai éprouvé cette difficulté en observant la préparation d'après laquelle j'ai exécuté la figure 30 (Pl. VIII). D'abord je l'ai observée au moment où elle était 
moins différenciée. Le cytoplasme au cours de ce stade se différencie avec difficulté, il est coloré en gris.

J'ai observé de petits et peu nombreux granules, colorés en noir, c'est à dire la partie du chondriome qui n'a pas subi les transformations décrites plus haut; on ne voit point de chondriome modifié. Après avoir différencié plus fortement la préparation, j'ai obtenu l'image décrite plus haut (fig. 30 Pl. VIII). Sur cette image apparaissent des éléments du chondriome colorés en gris, mais les grains minuscules, visibles avant la différenciation plus forte, se décolorent. Malheureusement, n'ayant pas fait auparavant de dessin de ces granules, j'ai été forcée, pour les rendre visibles, de faire le dessin d'une autre cellule dans le même ovaire et au même stade, seulement moins différenciée (fig. $31 \mathrm{Pl}$. VIII). Une preuve est irréfutable, c'est que beaucoup plus tôt (fig. 31 Pl. VIII), à côté des granules simples, apparaissent des formes de division du chondriome, en forme de biscuits et des granules liés entre eux à l'aide d'un filament gris, visible à une faible différenciation des préparations microscopiques.

A travers toutes les phases, décrites plus haut, c'est à dire dès le pachytène jusqu'au stade où la cellule-mère est développée, on ne voit pas de graisse sur les préparations osmiques. Ce n'est qu'à l'époque postérieure (fig. 32 Pl. IX) qu'on voit réapparaître une grande quantité de petites gouttes de graisse dans la cellule-mère, de même que dans les cellules des tétraspores qui dégénèrent (fig. 33 Pl. IX). Leurs formes correspondent à celles du chondriome dans l'intérieur duquel elles naissent. Je dois encore souligner qu'il y a une plus grande quantité de vésicules chondriomales et par conséquent plus de gouttes de graisse dans les cellules dégénérescentes de tétraspores que dans la cellule-mère (fig. 32 Pl. IX).

Malheureusement, la possibilité d'observations minutieuses de la transformation successive du chondriome se termine au stade de la cellule-mère, car le sac embryonnaire se contracte au cours de la fixation, il ne se colore pas bien et se déchire pendant qu'on le coupe.

Si j'ai réussi à obtenir des préparations des stades postérieurs du sac embryonnaire, ce n'est que pour le sac à deux et à cinq noyaux ( 3 noyaux du sac à 4 noyaux ne se sont pas encore divisés, tandis que l'un d'eux s'est déjà divisé). 
Dans le sac à deux noyaux on voit encore ces mêmes relations que dans la cellule-mère. Ainsi donc sur les préparations fortement différenciées on voit de grands anneaux colorés en gris (fig. 34 Pl. IX). Sur celles, qui sont au contraire moins différenciées il y a de petits grains noirs, (fig. 35 Pl. IX) liés pour la plupart deux par deux à l'aide d'un filament gris.

Dans les figures 31 (Pl.VIII) et 35 (Pl. IX) on observe une polarité dans la disposition du chondriome qui ne se distinguait pas jusqu'ici, le. chondriome étant beaucoup plus nombreux dans le pôle micropylaire du sac embryonnaire.

Je trouve la confirmation de ces rapports au stade du sac à cinq noyaux (fig. 37 PI. IX) où la forme vésiculeuse du chondriome, qui est la preuve d'une phase nouvelle de graisse, n'occupe que le pôle décrit plus haut. Dans la partie restante apparaissent de petits grains peu nombreux et colorés en noir. Sur la préparation osmique (fig. $38 \mathrm{Pl}$. IX) qui correspond à celle de la fig. 37, (PI. IX) les gouttes très nombreuses de graisse n'occupent que la partie du sac décrite plus haut, tandis que sur le pôle opposé elles apparaissent en très petite quantité.

En résumé on peut observer trois périodes dans l'apparition de la graisse dans le chondriome au cours de l'évolution du sac embryonnaire de l'Orchis latifolius L. De ces trois périodes j'ai pu étudier deux complétement, quant à la troisième, je n'ai pu observer que la phase initiale (les grains minuscules en division) et la phase terminale (au stade des vésicules et à celui des gouttes de graisse).

La première phase de l'apparition de la graisse a lieu dans la cellule-mère des mégaspores à la période d'accroissement intense de la cellule, au commencement de la prophase, au stade de longue durée de syni ‘esis (fig. 11, 12, 13 Pl. VII).

Au commencement du pachytène, la graisse se dissout et à partir de ce moment les petits grains peu nombreux du chondriome restant, commencent à se multiplier. En même temps se succèdent immédiatement deux divisions du noyau, aboutissant à la formation de la tétrade, après quoi vient le stade d'un plus long repos. C'est à cette époque que grandit la cellule-mère et dans le chondıiome, qui ayant traversé auparavant une évolution décrite plus haut, la graisse réaparaît ensuite (fig. 32, 33 Pl. IX). Cette graisse n'est pas aussi promptement consommée comme cela a lieu dans la cellule 


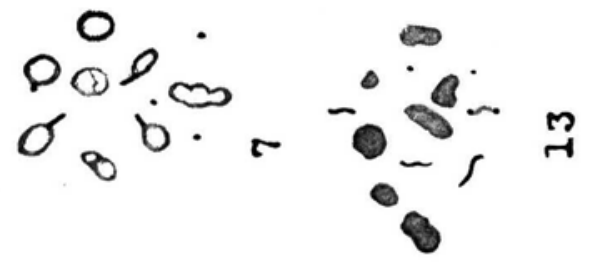

$\begin{array}{cc}0 & \\ .0 & 0 \\ 0 & 0\end{array}$

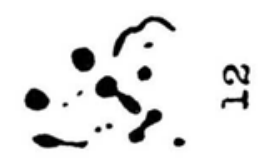

م

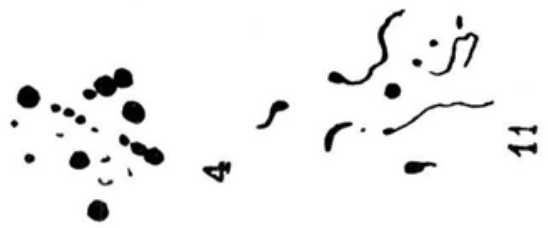

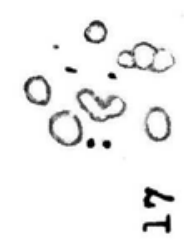

$$
0^{\circ}
$$
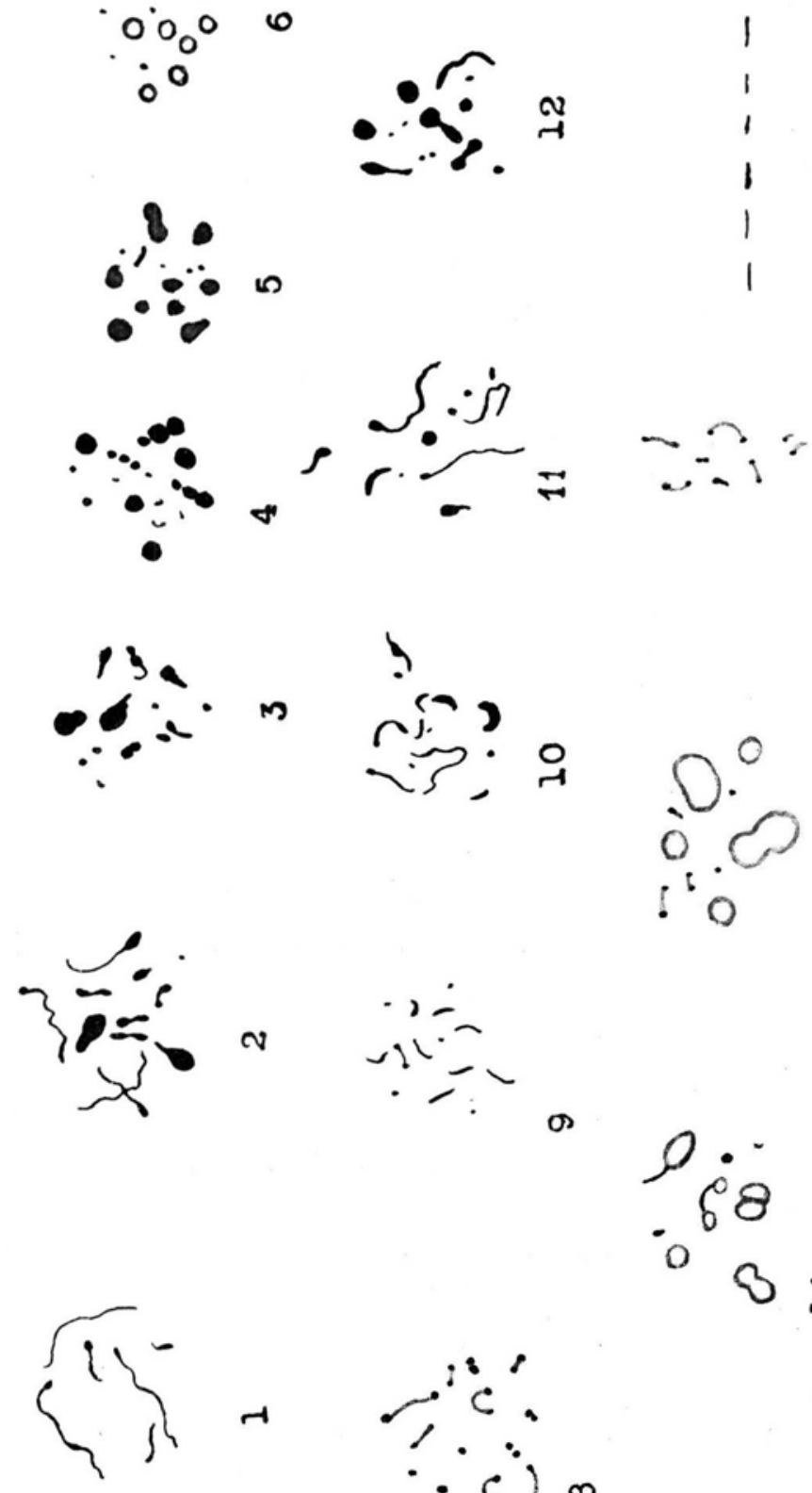

苞 
primordiale, car il y a encore dans le sac à deux noyaux des vésicules remplies de graisse, bien que ces vésicules soient moins nombreuses (fig. 3' $\mathrm{Pl}$. IX). D'autre part, cette division des noyaux s'effectue probablement beaucoup plus vite que le passage d'un stade, de la prophase de la mitose hétérotypique, à un deuxième, car je n'ai pas réussi à observer les stades de la division de la cellule-mére, bien que j'aie fixé une très grande quantité de matériel; tandis qu'en faisant des préparations sur les ovaires jeunes, j'ai pu, au contraire, observer très souvent le stade synizesis.

Le résidu du chondriome, c'est à dire celui en forme de grains petits et peu nombreux (fig. 31 Pl. VIII, 35 PI. IX), recommence á se multiplier, après quoi la graisse réaparaît en abondance (fig. 37, 38 Pl. IX).

Je donne ci-dessous (Fig. 178 du texte) le schéma de l'evolution du chondriome, observée par moi, afin d'en rendre plus clair son caractère. J'ai y omis des rapports quantitatifs du chondriome et je n'indique, que les transformations morphologiques.

\section{III. Étude vitale et postvitale.}

Mon étude a porté sur l'ovaire des bourgeons non encore épanouis et sur celui des fleurs, qui venaient de s'épanouir. Je procédais de la manière suivante: d'abord je coupais l'ovaire verticalement; tout de suite après, à l'aide d'une aiguille, je séparais les ovules et je les plongeais immédiatement dans une goutte de liquide où se trouvait le colorant donné. Pour protéger l'objet contre la pression mécanique, je munissais les quatre coins de la lamelle de supports de cire. Pour la coloration vitale, j'appliquais avec succès le vert Janus et la phénosafranine. Le violet de Dahlia ne donnait pas de résultats positifs.

En appliquant le vert Janus pour les concentrations de $1 / 30.000$ à $1 / 10.000$ sur le chondriome, je colorais en même temps les vacuoles au moyen du rouge neutre.

Pour faire la solution des colorants, j'employais de l'eau distillée additionnée de $0,75 \mathrm{NaCl}$ pour $100 \mathrm{~cm}^{3}$, contrairement à la majorité des auteurs, qui employaient la solution du saccharose.

Je colorais la graisse au Soudan III, et au liquide „Nadi”. 
J'ai fixé la concentration convenable du rouge neutre par voie d'essai. En appliquant le vert Janus en concentration $1 / 30.000$, les grains du chondriome se coloraient en verdàtre uniquement dans les cellules épidermiques, qui entouraient la cellule primordiale (fig. $39 \mathrm{Pl}$. IX). Cette opération répétée plusieurs fois et toujours avec le même résultat, m'a amenée à la conclusion, qu'avec la concentration aussi faible tout le colorant est absorbé uniquement par le chondriome des cellules épidermiques, alors qu'il ne pénetre pas dans la cellule-mère de mégaspores. Ce n'est qu'à la concentration de 1/10.000 que j'ai obtenu une très jolie coloration du chon. driome de la cellule primordiale (fig. 40 Pl. IX). Mais au bout de trois quart d'heurs se font remarquer dans le chondriome des changements bien visibles: le chondriome, qui apparait aux premiers moments de l'expérience sous forme de grains minuscules se renfle, grandit, devient très refringeant et vésiculeux. Peux de temps après survient la mort de la cellule. Ce fait est conforme aux résultats généraux obténus par Guilliermond (15), avec le même colorant vitale.

Aux stades les plus précoces le chondriome apparait sous forme de mitochondries granuleuses. Ce n'est qu'après avoir réussi à abréger le temps de la durée de la manipulation précédente l'observation vitale, qne j'ai commencé à apercevoir la présence dans la cellule-primordiale et dans les cellules épidermiques de nombreux et très minces chondriocontes. Cependant au bout de quelques minutes, ces filament commencent à subir une fragmentation dont résulte la formation de petits grains placés l'un à côté de l'autre (fig. $42 \mathrm{Pl}$. IX), et par suite de leur dissociation, apparaissent des grains dispercés sans aucune ordre (fig. 41, $42 \mathrm{Pl}$. IX).

A. Luxemburg $(22$, p. 369) à la suite de ses observations vitales sur le chondriome dans les cellules-mères du pollen des Malvacées, interprète d'une autre façon les granules rengées en forme de chaînette. L'auteur trouve que cettes chaînettes se forment plutôt par agglomération des granules particuliers que par la fragmentation des chondriocontes. Elle ne les a pas vus vivants. C'est pour cette raison qu'elle considère les éléments filamenteux et ceux en forme de bàtonnets, apparaissant sur la préparation fixée d'après la methode de Benda, comme de simples faits artificiels. 
Le chondriome, qui se trouve dans la cellule primordiale de l'Orchis latifolius L. entoure dans la plupart des cas le noyau d'une sorte d'anneau (fig. $44 \mathrm{Pl}$. IX), ou il s'amasse a côté de l'un ou de l'autre pôle du noyau (fig. 40, 41, 43 Pl. IX), tandis que dans les cellules épidermiques il occupe en générale une partie du cytoplasme près des parois inférieures (fig. $41 \mathrm{Pl}$. IX).

Comme on ne trouve pas sur les préparations fixées de telles régions séparées, je me crois autorisée à supposer, qu'un tel grouppement du chondriome doit être considéré comme résultat des déplacements par suite de la formation de la vacuole et des courants occasionés par la pénétration du colorant.

En observant des stades postérieurs, on aperçoit sur le terrain de la cellule primordiale, à côté des granules du chondriome, colorés au vert Janus, des gouttes plus grandes incolores et fortement réfringeantes (fig. 44, $46 \mathrm{Pl}$. IX). Ces gouttes donnent une réaction de la graisse (fig. 45 PI. IX).

Elles se colorent en rouge-brique à l'aide du Soudan III à chaud et en bleu à l'aide du mélange "Nadi".

A mesure qu'augmente la quantité de gouttes de graisse, diminue en même temps le nombre de granules qui se colorent au vert Janus (fig. 44, 46 PI. IX). Cette dépendance confirme l'observation exacte sur des matériaux fixés, concernant la formation de la graisse à l'intérieur du chondriome.

Au cours de la coloration vitale, je n'ai pas réussi à observer les parois du chondriome autour des gouttes de graisse. Il est extrêmement difficile d'aperçevoir une pellicule à la surface de la goutte réfringeante, même si cette pellicule est colorée. Je crois pourtant que le chondriome, observé au stade, où sur les préparations il se colore très faiblement en gris à l'hématoxyline, cesse aussi de se colorer au vert Janus.

Cela est d'autant plus possible, que pour les plastides, qui produisent de l'amidon et qui en général se colorent intensivement à l'hématoxyline, Guilliermond (15) a constaté ce même comportement à l'égard du vert Janus.

Cependant j'ai réussi à démontrer l'existance de la vésicule chondriomale autour des gouttes de graisse et voila par quels moyens j'y suis parvenue: en agissant sur les cel- 
lules avec le chloroforme, l'éther, l'alcool absolu et d'autres, j'observais les gouttes de graisse se dissoudre rapidement, mais en même temps le protoplasme commençait à ce contracter à tel point qu'il m'était impossible de continuer les observations quelconques sur la très petite cellule primordiale. Pour y arriver malgré tout, j'ai choisi des ovules dont les intéguments étaient plus développés; j'en ai pris ensuite une cellule de dimension plus grande et remplie de gouttes de graisse, et, en l'observant toujours au microscope, je mettais avec précaution quelques gouttes d'alcool absolu sous la lamelle en enlevant en même temps l'eau au moyen de papier buvard. Les gouttes de graisse se dissolvaient en quelques secondes. Mais a mesure qu'elles diminuaient, leur surface se contractait et se froissait, ce qui rappelait le refrognement du ballon au moment où l'air s'en dégage. Après la dissolution complète de la graisse, on voyait, à l'éndroit où il avait la goutte, une pellicule chondriomale froncée et rétractée.

Au cours de la coloration au vert Janus, je n'ai pu observer de formes transitoires entre les filaments et les vésicules remplies de graisse. Il en faut peut être chercher la cause dans ce que le chondriome en s'altérant les chondriocontes subissent une fragmentation et un renflement. Je dois aussi ajouter que mes observations vitales ont été exécutées à un grossissement beaucoup plus faible (que celles sur les matériaux fixés) auquel il m’a été impossible de faire réssortir les détails morphologiques des éléments particuliers du chondriome.

A la fin j'ai appliqué également la fénosafranine dans les concentrations établies par voie d'essais. Ainsi, après avoir plongé l'objet d'observation dans du colorant, j'ai pu d'abord observer les membranes se colorer en rouge, le cytoplasme en rose et enfin les grains du chondriome en rouge. En appliquant le violet de Dahlia, dont se servaient plusieurs auteurs pour la coloration vitale du chondriome, je n'ai pas réussi à obtenir de résultats positifs.

\section{Con c l us ions.}

Comme je l'ai déjà fait remarquer plusieurs fois, l'apparition de la graisse, au cours des stades déterminés du développement du sac embryonnaire, a lieu à l'interieur du 
chondriome qui subit d'abord certaines modifications morphologiques et ensuite commence à se colorer en gris à l'hématoxyline. C'est le stade qui précède immediatement le moment de l'apparition des gouttes "de graisse. Celle-ci crôt avec une vitesse extrême, de sorte que bientôt après sa différenciation elle remplit tout l'intérieur des éléments du chondriome. Par conséquence le chondriome prend la forme des vésicules à parois minces, après quoi les pellicules chondriomiques ainsi que les goutles de graisse disparaissent.

On peut admettre facilement qu'on a affaire ici avec le chondriome ,actif dans la photosynthèse ou plastes”, dans le sens déterminé par Guilliermond $(8,9,10,18,19$ et d'autres), c'est à dire avec le chondriome, qui dans notre cas produit de la graisse, ou pour mieux dire avec les oléoplastes, et que par conséquent la graisse se trouve dans le mêne rapport à la photosynthèse, que l'amidon. En faveur de cette affirmation, plaiderait le fait, que les globules de graisse constituent l'unique substance visible du métabolisme aussi dans les parties vertes (feuilles, tiges, parties vertes de l'ovaire), où ces globules apparaissent dans des chloroplastes, qui à leur tour subissent les mêmes transformations, que le chondriome des cellules embiyonnaires. Il est à discuter si le chloroplaste, après avoir produit de la graisse, subit le mème épuisement et la même dégénérecence, car il m'a été impossible de le constater. Pourtant certaines de mes observations font supposer que cet état de choses existe en réalité.

D'autre part en faveur de la lipophanérose de Guilliermon d $(20$, p. 570$)$ plaide avant tout le fait, qu'après le détachement de la goutte de graisse, le chondriome est détruit, tandis qu'après la production de l'amidon et des pigments, d'après les observations faites par Guilliermond, Mangenot et Emberger (2), (3) il reprend son état initial (la réversibilité fonctionelle du chondriome).

De plus, en faveur de la lipophanérose plaide aussi un autre fait, qu'après l'apparition de la graisse, la pellicule chondriomale se colore si faiblement, qu'il est difficile de constater sa présence et de la rendre visible. Ce fait peut ètre considéré comme le résultat de la séparation du complexe lipoprotéique, qui constitue le chondriome, en la pelli- 
cule protéique et en élément lipoidique, qui s’accumule sous la forme de gouttes a l'intérieur de la dite pellicule.

Les transformations, que subit le chondriome avant et au cours de l'apparition de la graisse, comme je l'ai déja observé, sont à peu près identiques avec celles qu'avait observées Nadson dans son ouvrage de 1926 (28).

Mes observations m'ont permis de constater en outre que dans la cellule embryonnaire la graisse apparait périodiquement avant, ou au commencement de la division et y est tout de suite dissoute.

Le chondriome qui se trouve dans les cellules dégénérescentes des tétraspores subit néanmoins les mêmes transformations, qu'il avait subi dans la cellule primordiale pendant la période de la formation de la graisse et qu'il subit exactement au même moment dans la cellule mère du sac embryonnaire. Il faut noter, qne dans la cellule-mère la graisse se produit en quantité plus petite. Une partie du chondriome (très petite du reste), celle en forme de petites plaquettes et de grains minuscules y reste, se multiplie et, dans la phase de la production de la graisse, subit progressivement les transformations décrites plus haut.

Dans les cellules dégénérescentes, au contraire, la graisse se forme en plus grande quantité et probablement le chondriome tout entier est détruit au cours du processus de la production de la graisse. Il est d'autant plus difficile d'observer ce fait que le terrain entier du cytoplasme est rempli de gouttes de graisse et que les cellules dégénérescentes se différencient avec difficulté.

En ce qui concerne les facteurs, qui provoquent la lipophanérose, il faut admettre que le plus probablement ils sont plusieurs: ce serait des symptomes, ou de nature patologique, ou de dégénérescence, ou au contraire le résultat d'une activité progressive de la cellule. Dans ce dernier cas on pourrait interpréter ce fait comme conséquence de la production intense d'une substance lipoïdique dans le corps du chondriome, substance qui trouble l'équilibre quantitatif du complex lipoprotéique du chondriome, qui a existé jusqu'ici, et qui de cette façon provoque la séparation de cette substance en élément lipoïdique et en élément protétique.

Cette interprétation n'excluerait pas celle de la lipopha nérose au cours des observations de Mangenot (25) sur 
le rôle de la lumière sur la formation des gouttes de graisse dans des chloroplastes.

La lipophanérose dans le processus de l'apparition des gouttes de graisse dans le chondriome justifie, au moins en théorie, la possibilité de l'apparition des gouttes de graisse (lipoïdiques) directement dans le cytoplasme, comme l'a constaté Guilliermond (11, p. 65; 14, 15), Emberger (1, p. 344), Mangenot (23, p. 982) et autres. Cela s'explique par le fait, que le cytoplasme, étant, comme le chondriome, une substance lipoprotéique (seulement dans une proportion différente) pourrait subir, dans certains cas, une lipophanérose.

En résumant les résultats exposés plus haut je suis en droit de conclure, que, quant à l'emploi du terme ,oléoplaste“ et par conséquent, quant à l'affirmation de l'identité du processus de la formation de la graisse avec celui de la production de l'amidon, de la chlorophylle de la xantophylle et c. exclusivement dans les plastes, il faut pour le moment garder une certaine reserve.

Il s'agit maintenant de savoire à quelle catégorie du chondriome nous avons à faire au cours du développement du sac embryonnaire de l'Orchis latifolius L. Les résultats de mon travail tendent à indiquer, que le chondriome tout entier subit progréssivement les mêmes transformations. C'est ì dire qu'après la dissolution de la graisse et des vésicules chondriomiques, les granules peu nombreux du chondriome qui restent jusqu'a présent passifs se multiplient et subissent plus tard les mêmes transformations, tandant à la production de la graisse et à leurs complètes destruction finale. Les granules peu nombreux du chondriome résiduel commencent à se multiplier à leur tour et ainsi de suite.

Dans la cellule dégénérescente des tétraspores, tout le chondriome semble au contraire prendre la forme de gouttes de graisse.

Si l'on compare maintenant ces observations avec opinion émise par Guilliermond dans son travail de 1925 (18, p. 26$)^{1}$ ) au sujet de la dualité du chondriome, le travail pré-

1) „On voit apparaitre les deux variétés dont l'une correspond aux plastes et a pour fonction produire de l'amidon, de la graisse, de la chlorophylle et des pigments xantophylliens et carotiniens et dont l'autre se montre inactive dans ces phénomènes et a rôle encore inconnu". 
sent constaterait la possibilité de la transformation du chondriome entier de la cellule végétale en plastes. Chaque élément chondriomal de la cellule végétale aurait le même potentiel fonctionel et par conséquent il confirmerait ,l'unité du chondriome" de Motte (25) dans la cellule végétale.

On pourrait supposer, que cette unité ne peut se révéler dans le cas, où se produit l'amidon et le pigment en égard à „La réversion des plastes" d'E $\mathrm{m}$ b e r g e r (2). Dans notre cas, an contraire, gràce à ce que les plastes, dans lesquelles a pris naissance la graisse, dégénèrent, le potentiel propre au chondriome résiduel, qui n'a pas pris part dans la phase précédente de l'apparition de la graisse, (peut-être soumise à l'influance de certins facteurs régulateurs) se révèlent tout à fait distinctement. L'interprétation émise ci-dessus purrait être considérée comme étant conforme aux théories de Meves, de Motte (25) ainsi qu'aux observations de A. L u x e m bu r g (22, p. 388).

Mais comme nous n'avons pas aujourd'hui de connaissance fixes sur les processus liés avec l'apparition de la graisse, il est nécessaire de tenir compte d'une autre possibilité. À savoir si l'on admet, comme je l'ai déjà dit plus haut, que la production de la graisse peut être provoquée par un processus autre, que cellui de la production de l'amidon, de la chlorophylle et du pigment (lipophanérose de Guilliermond 20, p. 570), ce processus cesserait alors d'être le criterium permettant de distinguer le chondriome ,actif" de "l'inactif“ dans la photosynthèse. En faveur de cette thèse plaiderait la production de la graisse dans le chondriome de la cellule animale (Altman et Metzner, Arnold, L. et R. Zoja, O. et R. Van der Stricht, Lams, Van Darme, Bluntschi, Russo, M-lle Loyer, Regaud, Dubreuil, Luna, Hoven, Athias - cités dans le travail de R. Noël (27), ainsi que Landreyt (21), Noël (27) et Maximow (24)) dans laquelle on n'accepte que l'existence du chondriome ,inactif“"

En présence de ces dernières considération, mes observations ne serait pas en principe contradictoires avec les opinions sur l'existence des deux catégories du chondriome dans cellule végétale.

Pour ce qui est de la multiplication des éléments chondriomaux, on doit faire remarquer que pendant la période du développement du sac embryonnaire de l'Orchis latifolius $L$. 
on peut observer, comme je viens de le dire, les moments d'un accroissement assez rapide de cet élément du cytoplasme.

En faisant tour à tour la comparaison des figures 18, 19, 20, 21 et $22-\mathrm{Pl}$. VIII, on voit distinctement, au cours de ces stades, une augmentation du nombre des grains minuscules qui représentent le chondriome.

Après l'apparition de la graisse dans le chondriome, une quantité plus grande de ce chondriome disparait (c'est le stade de la synizèse, ou les premiers moments du pachytène) et il ne reste alors que des grains minuscules dans le cytoplasme. Dans l'espace de temps qui est compris entre pachytène et la diakinèse on voit une augmentation rapide du nombre des grains. Leurs dimensions sont en moyenne les mêmes que celles des granules peu nombreux aux premiers stades du pachytène; en tout bas ils ne sont pas plus petits.

Le trait caractérictique pour cette periode est la grande quantité de petits grains liés entre eux deux par deux, à commencer par ceux, qui se touchent directement, (ce qui leur donne une forme de biscuits) jusqu'à ceux qui sont unis à l'aide d'un court filament coloré en gris. Ce filament atteint souvent une longueur assez considérable. C'est aussi dans les dyades, qu'on rencontre des grains lié de cette même façon, où à côté de ces éléments granuleux commencent à apparaître des chondriocontes sous la forme de courts filaments et de bàtonnets.

D'après Wagner (34), la disposition des grains liés à l'aide d'un très mince filament coloré en gris, pourrait être interprétée comme celle du chondrioconte, qui réaparaît ,,de novo" dans le cytoplasma et qui commence à se colorer fortement à ses extrémités. Mais il faudrait alors chercher des formes transitoires, c'est à dire celles en forme de courts et noirs filaments liés au milieu à l'aide d'un segment, qui se colore faiblement. Il serait aussi difficile d'expliquer l'augmentation du nombre des grais particuliers.

On observe ces-mêmes grains et ces-mêmes biscuits pour la seconde fois dans la cellule-mère du sac embryonnaire après la seconde phase de l'apparition de la graisse, ainsi que dans le sac à deux noyaux.

Toutes ces données font supposer, conformement à l'opinion de la plupart des cytologistes contemporains (voir Guilli e rmond 18, p. 147), qu'on a affaire à la multiplication du 
chondriome par voie de division et que les grains liés entre eux constituent un stade transitoire, au cours de la division du chondriome, entre le stade correspondant au chondriome en forme de biscuits et deux grains libres.

Ce travail a été exécuté au Laboratoire de Botanique Générale de l'Université de Varsovie sous la surveillance bienveillante de Monsieur le prof. Zygmunt Wóycicki. Je me fais l'agréable devoir de lui exprimer ici mes remerciements les plus sincères pour ses précieux conseils, qu'il a bien voulu me fournir au cours de mes récherches. Je dois également une vive reconnaissance à Monsieur le dr. W. A. B e cker, auprès de qui j’ai toujours trouvé, au cours de mon travail, des encouragements et de très utiles indications.

\section{Explication des figures.}

Toutes les figures se rapportent aux stades particuliers du développement du sac embryonnaire de l'Orchis latifolius L. Elles ont été exécutées a l'aide de l'appareil à dessiner d'Abbe. Pour exécuter celles de 1 à 36, j'ai fait usage de l'objectif à immersion $1 / 12$ de Reichert et de l'oculaire 25 au grossissement 2500 fois environ. Les préparations microscopiques on été fixés d'aprés la méthode de Lewitsk y.

Préparations oxygénées et colorées à l'hématoxyline ferrique de Heidenhain. Dans toutes les figures sauf la figure 10 il $y$ a des coupes verticales de l'ovaire.

\section{PLA N C H E VII.}

Fig. 1. Stade le plus précoce de la cellule primordiale du sac embryonnaire à l'état initial. Chondriome principalement filamenteux.

Fig. 2. Cellules épidermiques de l'ovule avec des chondriocontes filamenteux (stade correspondant à celui de la fig. 1).

Fig. 3. Fragment de l'ovule avec la cellule primordiale à l'époque d'accroissement. Le chondriome se gonfle et subit une fragmentation. Noyau à l'état de repos.

Fig. 4. Fragment de l'ovule avec la cellule primordiale. A còté des chondriocontes filamenteux, ont voit des formes transitoires des spermatozoïdes et celles des têtards. Noyau à l'état de repos. Dans les cellules épidermiques apparaissent des grains qui constituent la coupe transversale des éléments filamenteux. 
Fig. 5. Cellule primordiale.Le noyau est au stade de leptotène et la structure n'est pas prise en considération. On voit une quantité de moins en moins petite de filaments dans le chondriome; une quantité de de plus en plus grande de formes en tètards, de petites plaquettes gonflées à deux extrémités ainsi qu'une quantité de grains aux dimensions considérables.

Fig. 6. Cellule primordiale. Le chondriome est représenté par les mêmes formes que cellui de la figure précédente. On remarque le manque total d'éléments filamenteux.

Fig. 7. Cellule primordiale. La structure du noyau n'est pas prise en considération. Stade du zygotène. Le chondriome à peu près granuleux. A côté de lui ont voit une petite quantité d'anneaux du chondriome, colorés en gris, dans lequel s'est distinguée la graisse.

Fig. 8. Cellule primordiale. La structure du noyau n'est pas prise en considération. Stade du zygotène. A côté des éléments du chondriome, décrits plus haut, on voit des grains augmentés se colorant faiblement en gris.

Fig. 9. Cellule primordiale. Commencement de synizesis. On voit une quantité de plus en plus grande de grains du chondriome coloré en gris.

Fig. 10. Coupe transversale de l'ovule et de la cellule primordiale. La plupart du chondriome constituent des grains aux dimensions considérables, colorés en gris, de petits grains colorés en noir et des plaquettes peu nombreuses. Quelque'uns des éléments en tètards ne sont pas encore transformés,

Fig. 11. Cellule primordiale. Stade de synizesis. Le chondriome constituent: des anneaux colorés en gris (vésicules remplies de graisse), ainsi que des grains minuscules et des plaquettes colorées en noir très peu nombreuses.

Fig. 12. Cellule primordiale. Le mème stade que celui de la figure 11. Le chondriome constituent: des anneaux simples, des anneaux liés entre eux, les éléments en forme de raquettes de tennis (tous ces éléments sont plus gros par rapport à ceux de la fig. 11), ainsi que des grains minuscules colorés en noir et des plaquettes très peu nombreuses.

Fig. 13. Cellule primordiale. Le chondriome est représenté par les mèmes formes que celles de la fig. précédente. Le contour des anneaux devient de plus en plus faible.

Fig. 14. Stade correspondant à celui de la fig. 11. On y voit de petites gouttes de graisse peu nombreuse et colorées en brun à l'osmium ${ }^{1}$ ).

Fig. 15. Cellule primordiale. Stade correspondant à cclui de la fig. 11. Les globules de graisse sont plus gros et plus nombreux. Ils sont colorés à l'osmium ${ }^{1}$ ).

Fig. 16. Cellule "primordiale. Stade correspondant à celui de la fig. 12. Gouttes de graisse plus grosses, et colorées à l'osmium ${ }^{1}$ ).

Fig. 17. Cellule primordiale. Stade correspondant plus ou moins à celui de la fig. 13. De grosses gouttes de graisse ${ }^{1}$ ).

1) Les figures: 14, 15, 16, 17 ont été exécutées d'après les préparations non oxygénés et non colorées à l'hématoxyline ferrique: 


\section{P L A N C H E VIII.}

Fig. 18. Cellule primordiale. Stade du pachytène. Il n'est pas tenu compte du noyau. Le chondriome constituent: des granules particuliers peu nombreux, colorés en noir à l'hématoxyline, des éléments en forme de biscuits et des granules liés à l'aide d'un filament plus court ou plus long.

Fig. 19. Cellule primordiale. Stade du pachytène. A còté des éléments du chondriome décrist plus haut, qui sont en nombre un peu plus grand, on voit un anneau coloré en gris.

Fig. 20. Cellule primordiale. Strepsitène. Le chondriome offre la mème structure que celui de la fig. 19, il n'est qu'un peu plus nombreux.

Fig. 21. Cellule primordiale. Dyacinèse. Le chondriome y est représenté par les mèmes formes que celui des figures précédentes avec cette différence seulement qu'il y est plus nombreux.

Fig. 22. Cellule primordiale. Métaphase de la mitose hétérotypique. Chondriome pareil à celui de la figure précédente. On le voit réparti uniformement dans le cytoplasme.

Fig. 23. Cellules de la dyade immédiatement après la mitose. Chondriome reste le mème. Quantité de grains liés, noins nombreuse.

Fig. 24. Cellules de la dyade. Chondriome représenté en majeure partie par de petites mitochondries granuleuses. Grains liés sont très peu nombreux. Les chondriocontes réaparaissent sous la forme de bâtonnets courts et colorés en noir.

Fig. 25. Cellules de la dyade au stade de la mitose homéotypique. Grains moins nombreux que ceux de la fig. 2'. Chondriocontes plus nombreux et en forme de longs et de minces bâtonnets et de filaments onduleux et avee de petits renflements sur un ou plusieurs points de leur traget.

Fig. 26. Cellules de la dyade. La cellule interne de la dyade est au stade de télophase. Dans la cellule interne en dégénérescence on voit une plaque équatoriale. Le chondriome est en forme de tètards et de poires, de gros et petits grains et des bâtonnets courts et peu nombreux.

Fig. 27. Trois cellules de la tétraspore. Chondriome en général le mème que celui de la figure précédente.

Fig. 28. Cellule-mère. Stade de repos. Chondriome à peu près granuleux. Deux tétraspores dégénèrent. Tétraspore centrale colorée en noir. Tétraspore extérieure est au stade de l'anaphase. On y voit de gros grains du chondriome colorés en noir.

Fig. 29. Cellule-mère. Mème stade que celui de la fig. 28. Chondriome plus clair (visible) que celui de la figure précédente.

Fig. 30 et 31. Cellule-mère et deux tétraspores dégénérescentes. Fig. 30. Préparation fortement différenciée. Noyaux incolores. On n'apercoit qu'une partie du chondriome qui au cours de ce stade remplit la cellule-mère. Le chondriome est sous la forme d'éléments aux dimensions considérables, fortement renflés et colorés en gris. La fig. 31 représente des grains minuscules, des éléments en forme de biscuits et des grains liés entre eux qui constituent la seconde partie du chondriome, à savoir la partie moins nombreuse. On aperçoit tout cela sur la préparation plus fortement colorée. 
Fig. 32. Cellule-mère et tétraspores dégénérescentes. On y voit des anneaux très nombreux (surtout dans les tétraspores) et des éléments en forme de raquettes de tennis, colorés en gris.

Fig. 33. Cellule-mère et cellules dégénérescentes, remplies de gouttes de graisse. Cette dernière est colorés à l'osmium. Préparation non oxygénée et non colorée. Elle correspond à celle de fig. 32.

Fig. 3't Sac embryonnaire à deux noyaux. Cellules extérieures des tétraspores en état de dégénérescence. Elles sont toutes couvertes de l'hématoxyline. Préparation fortement différenciée. Il n'est resté du chondriome que des anneaux colorés en gris. Apparaissent des vacuoles.

Fig. 35. Stade correspondant à cellui de la fig. 3'. Préparation plus fortement colorée. On n'y voit plus d'anneaux, on voit au contraire de petits grains et des éléments pareils à ceux de la fig. 31. Dans la disposition des grains on voit une polarité.

Fig. 36. Sac embryonnaire à deux noyaux. Même stade que celui des figures 34 et 35 . Préparation non oxygénée et non colorée. On y voit des gouttes de graisse. Leur nombre dans le sac embryonnaire est beaucoup plus petit qu'il n'était dans la cellule-mère de la fig. 33 .

Fig. 37. Stade correspondant à celui de la figure précédente, colorée à l'hématoxyline. Le chondriome constituent des anneaux colorés en gris. Grossissement 1230 fois environ.

Fig. 38. Sac à cinq noyaux. La graisse est colorée à l'osmium. On voit une polarité dans la disposition des gouttes. Grossissement 1230 fois environ.

Fig. 39. Cellules épidermiques de l'ovule au stade précoce de la cellule primordiale. Coloration vitale au vert Janus de petits grains. Immersion $1 / 12$ de Reichert, oculaire 10, grossissement 1000 fois environ.

Fig. 40. Fragment de l'ovule avec la cellule primordiale augmentée. Dans les cellules épidermiques ainsi que dans la cellule primordiale on voit des grains du chondriome, colorés au vert Janus. Immersion 1/12 de Reichert, oculaire 10, grossissement 1000 fois environ.

Fig. 41. Fragment de l'ovule avec la cellule primordiale augmentée. Cellules épidermiques avec des vacuoles colorées au rouge neutre. Le chondriome constituent des grains minuscules colorés au vert Janus. Immersion $1 / 12$ de Reichert, oculaire 10, grossissement 1000 fois environ.

Fig. 42. Fragment de l'ovule. Cellules épidermiques. On n'y voit pas de membranes cellulaires. Vacuoles colorées au rouge neutre. Chondriome coloré au vert Janus, souvent il est rangé en forme de chaînettes. Immersion $1 / 12$ de Reichert, oculaire comp. 8, grossissement 1350 fois environ.

Fig. 43. Ovule avec la cellule primordiale augmentée. Vacuoles colorées au rouge neutre. Chondriome coloré au vert Janus. Immersion 1/12 de Reichert, oculaire 10, grossissement 1000 fois environ. 
Fig. 4'. Fragment de l'ovule. Dans les cellules épidermiques on voit des vacuoles colorées au rouge neutre. A còté du chondriome, coloré au vert Janus, on voit des gouttes de graisse incolores. Immersion $1 / 12$ de Reichert, oculaire comp. 8, grossissement 1350 fois environ.

Fig. 45. Cellule primordiale avec des gouttes de graisse colorées au Sudan III et avec des grains minuscules du chondriome. Immersion $1 / 12$ de Reichert, oculaire 10, grossissement 1000 fois environ.

Fig. 46. Cellule primordiale. Multitude de gouttes de graisse incolores. Petits grains du chondriome moins nombreux et colorés au vert Janus. Dans les cellules épidermiques on voit des vacuoles. Immersion $1 / 12$ de Reichert, oculaire 10, grossissement 1000 fois environ.

\section{Lit térature.}

1. E m b e r g e r L. „Observations cytologiques sur la bulbe de Lilium candidum L.." C. R. Ac. Sc., T. 179, 1924, p. 344.

2.

3.

4. Guilliermond $\Lambda$. „Sur la réversibilité des plastes chez les végétaux“. C. R. Ac. Sc., T. 181, 1925, p. 879 .

„Nouvelles recherches sur le chondriome de la cellule végétale“. Rev. Gén. Bot., T. 37, 1927.

„Nouvelles observations vitales sur le chondriome des cellules épidermiques de la fleur d'Iris germanica. No. 2. Production de globules graisseux au sein des mitochondries et des plastes. Fixation du chondriome". C. R. Soc. Biol. T. 78, 1915, p. 245.

5. „Sur le caractère du chondriome dans les premiers stades de la différenciation du sac embryonnaire de Tulipa suaveolens". C. R. Soc. Biol., T. 82,1919 , p. 976.

6. „Sur les éléments figurés du cytoplasme“. C. R. de Séances de l'Acad. des Sc., T. 170, 1920, p. 612. „Sur l'évolution du chondriome pendant la formation des grains de pollen de Lilium candidum". C. R. des Séances de l'Acad. des Sc., T. 170,1920 , p. 1003.

8. „Sur la coëxistence dans la cellule végétale de deux variétés distinctes de mitochondries". C. R. Soc. Biol., T. 83, 1920, p. 408.

9. „Nouvelles remarques sur la coëxistence de deux variétés de mitochondries dans les végétaux chlorophylliens". C. R. Soc. Biol., T. 83, 1920, p. 1046.

10. „Sur les caractères et l'évolution du chondriome dans les végétaux chlorophylliens". C. R. Soc. Biol., T. 84, 1921, p. 197.

11. „Sur les éléments figurés du cytoplasme chez les végétaux: chondriome, appareil vacuolaire et granulation lipoïdes“. Arch. Biol., T. XXXI, 1921, p. 1 
12. Guilliermond A. „Sur la formation des grains d'aleurone et de l'huile dans l'albumen de Ricin“. C. R. Soc. Biol., T. 86,1922 , p. 434 .

13.

14.

$\mathrm{J} 5$.

16.

17.

18.

19. "Sur l'origine et la signification des oléoplastes". C. R. Soc. Biol., T. 86, 1922 , p. 437.

„Remarques sur la formation des chloroplastes dans le bourgeon d'Elodea canadensis“. C. R. des Séances de l'Acad. des Sc., T. 175, 1922, p. 283. "Sur la coloration vitale des chondriosomes". C. R. Soc. Biol., T. 89, 1923, p. 527.

"Nouvelles observations sur l'évolution du chondriome dans le sac embryonnaire des Liliacées". C R. des Séances de l'Acad. des. Sc., T. 177, 1923 , p. 1138.

„Recherches sur l'évolution du chondriome pendant le développement des grains de pollen et du sac embryonnaire chez les Liliacées et sur la signification des formations ergastoplasmiques". Ann. Sc. Nat. Bot., T. 6, 48, 192 '

„La structure de la cellule végétale“. The memorial publication in honor of the 100th Birthday of J. G. Mendel issued by the Czechoslovac eugenics Society in Prague, 1925.

„Le chondriome de la cellule végétale. A propos d'un article récent de M. Pavillard“. Arch. Bot. Bull. Mens., T. 1, No. 8-9, 1927.

20. Guilliermond A., Mangenot G. et Plantefol L. Traité de cytologie végétale. Le François 1933.

21. Landreyt F. Le chondriome des cellules adipeuses". C. R. Soc. Biol., T. 82,1919 , p. 375 .

22. Luxemburg A. „Recherches cytologiques sur les grains de pollen chez les Malvacées“. Bull. de l’Acad. Pol. des Sc. et Let., Série B., 1927 , p. 363.

23. M a nge not G. "Sur les formations graisseuses des Vaucheria“. C. R. Soc. Biol., T. 83,1920 , p. 982 .

24. M a x i m o w. „Sur la structure des chondriosomes“. C. R. Soc. Biol., T. 79,1916 , p. 465 .

25. M o t t e J. "Contribution à la connaissance cytologique des Muscinées". Ann. Sc. Nat. Bot., 20, 298, 1928.

26. Nadson G. A. „Le chondriome est la partie la plus sensible aux rayons X“. C. R. Soc. Biol., T. 95, 1926, p. 378.

27. No ël R. „Sur un mode d'élaboration de graisse osmioréductrice dans la cellule hépatique də souris blanche“. C. R. Soc. Biol., T. 85, 19211922, p. 1930.

28. Orman E. „Recherches sur les différenciations cytoplasmiques“. La Cellule, T. 28, 1912.

29. Py G. „Recherches cytologiques sur l'assise nourricière des grains de pollen d'Helleborus foetidus, Euphorbia Santia et E. Pélus" C. R. Acad. Sci., 1929. 
30. P y G. „Recherches cytologiques sur l'assise nourricière des microspores et les microspores des plantes vasculaires“. Rev. Gén. de Bot., 't', Nr. 525, 1932.

31. V e r m o e se n C. „Contribution à l'étude de l'ovule, du sac embryonnaire et de la fécondation dans les Angiospermes". La Cellule, T. 27, 1911, p. 115.

32. Wa g n er N. ,Sur les chondriosomes et les plastides pendant la formation du pollen chez Veratrum album L. var. Lobelianum Ber“. Mém .Soc. Nar. Kiew., XXV, 1915.

33. - Evolution du chondriome pendant la formation des grains de pollen chez les Angiospermes“. Biol. Gener., Band. III., Heft 1/2, 1927.

3'. - „Sur la formation ,de novo“ des chondriosomes dans le cytoplasme des cellules-mères des grains de pollen chez les Angiospèrmes". Biol. Gener., Bd. III, H.3, 1927.

35. Wóy c i k i Z. „Sur les cristalloüdes de noyaux et les oleoplastes chez Ornithogalum caudatum“. Bull. Acad. Pol. Sci. et Let., 1929. 
M

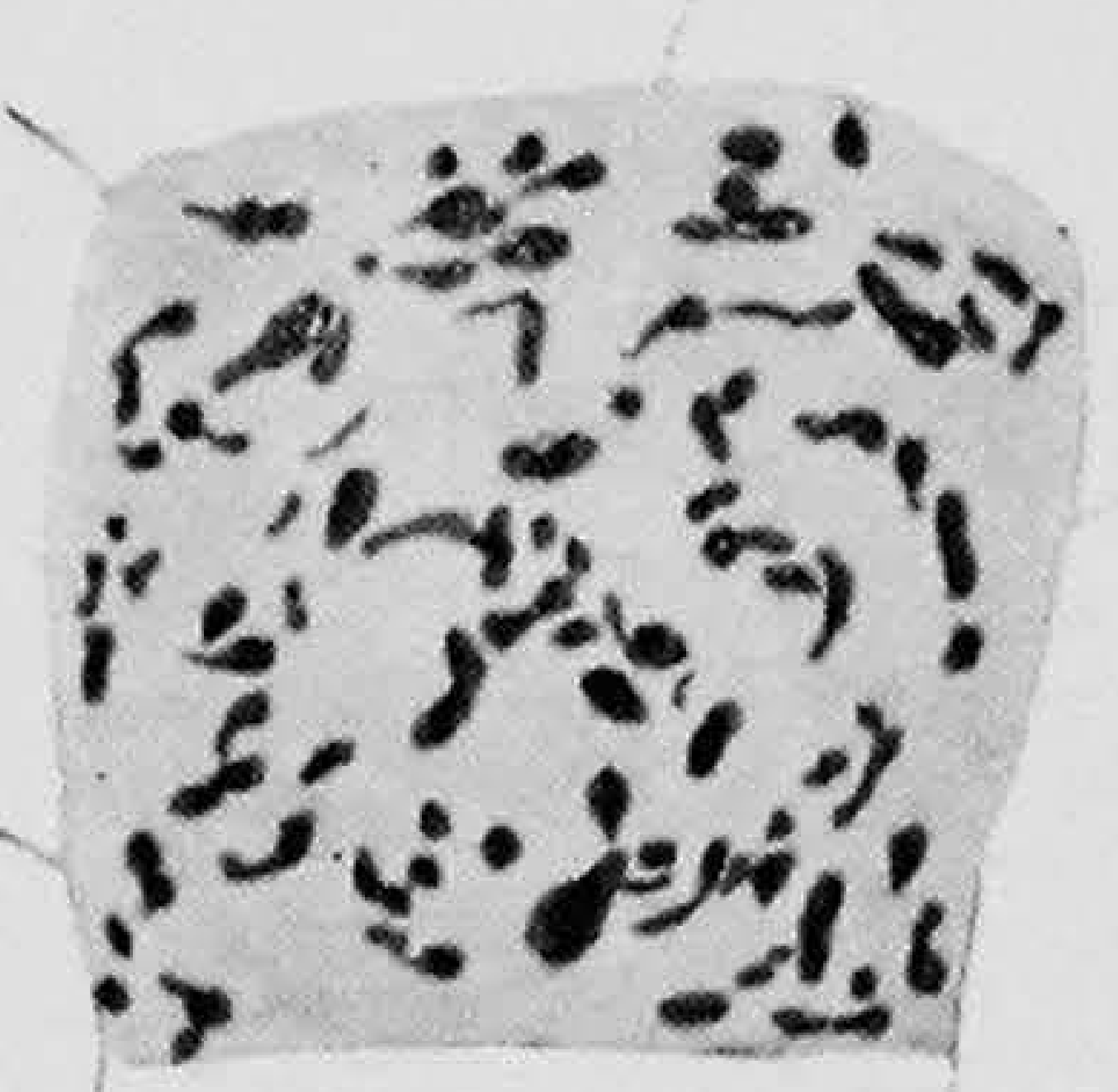

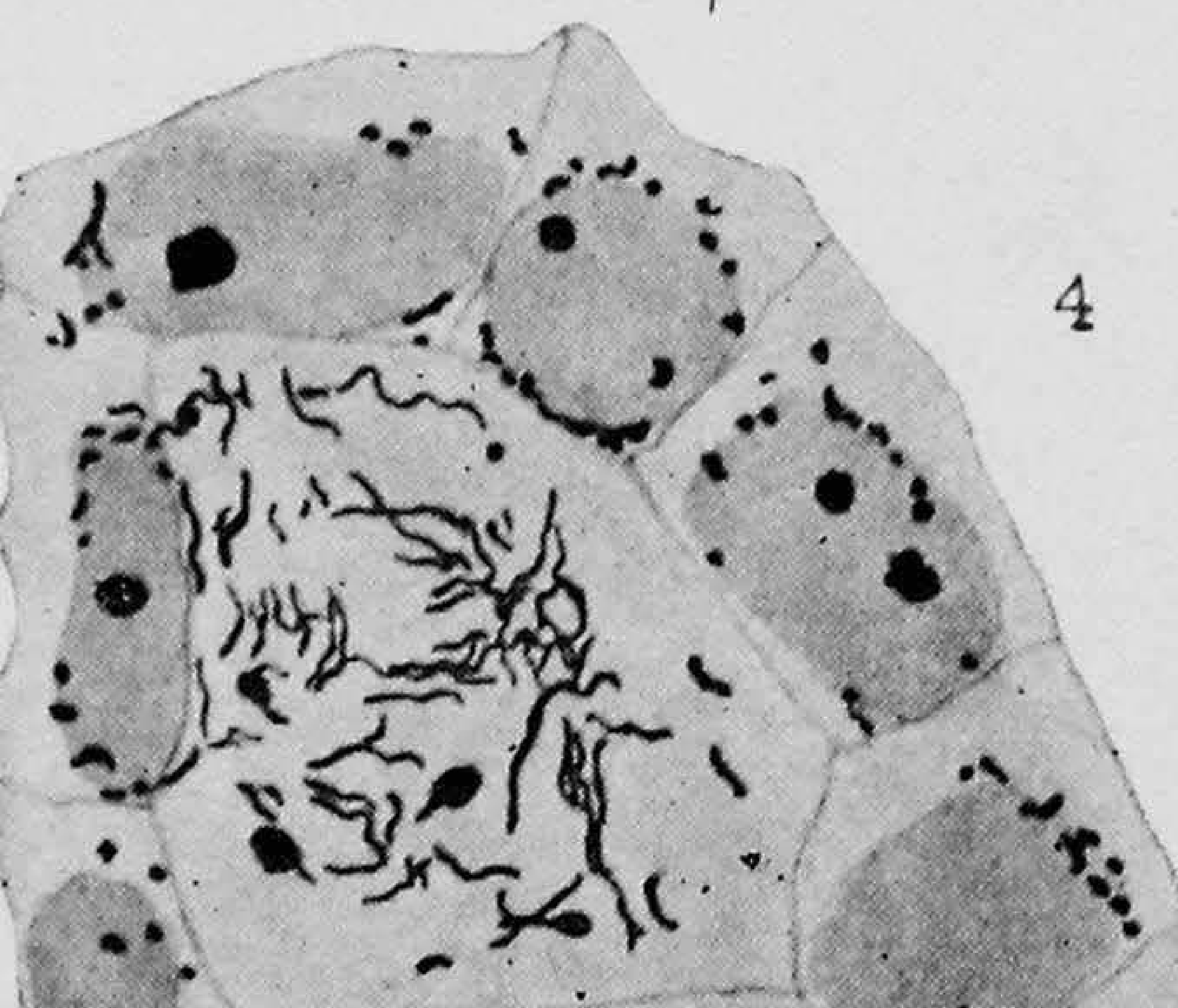
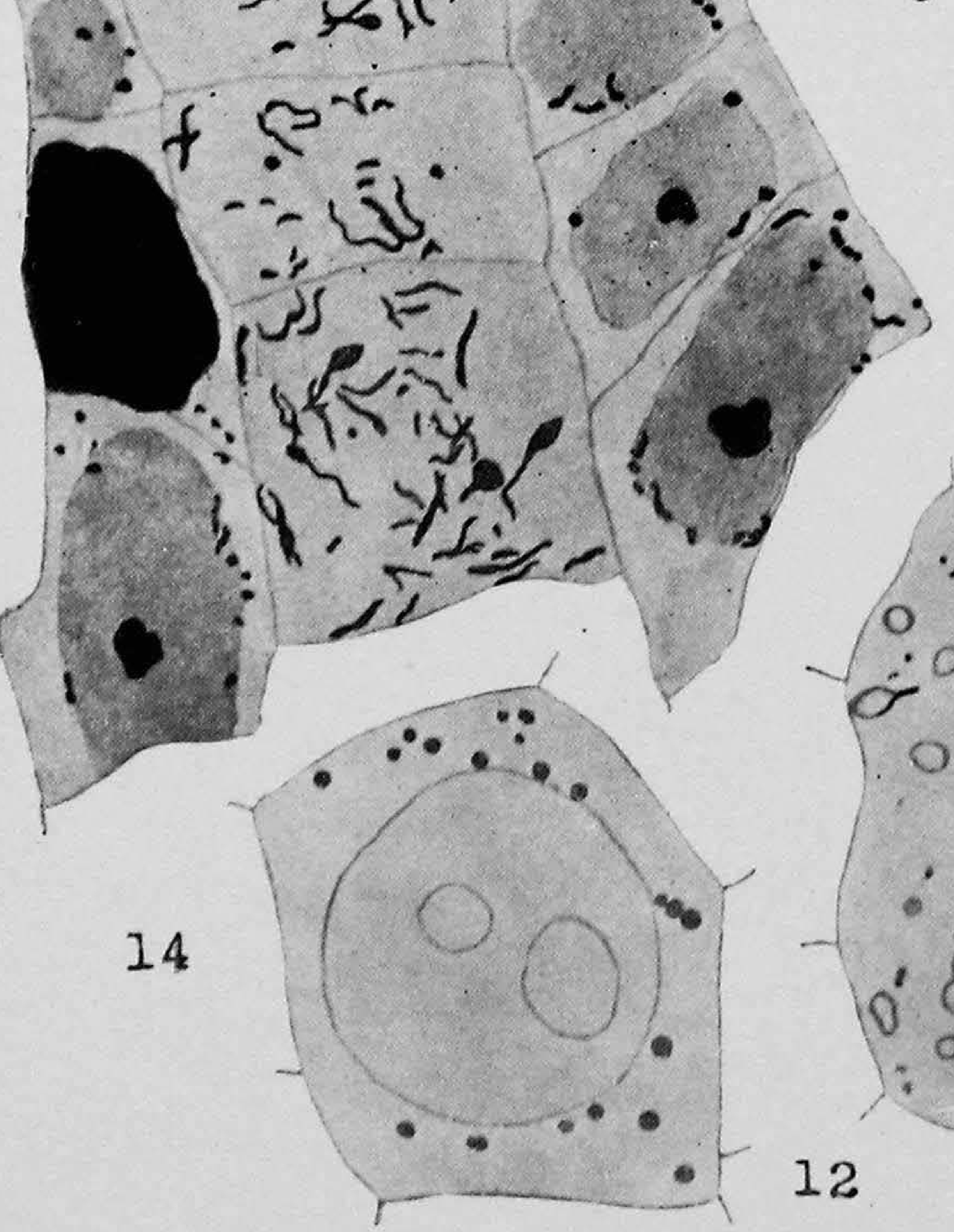

12

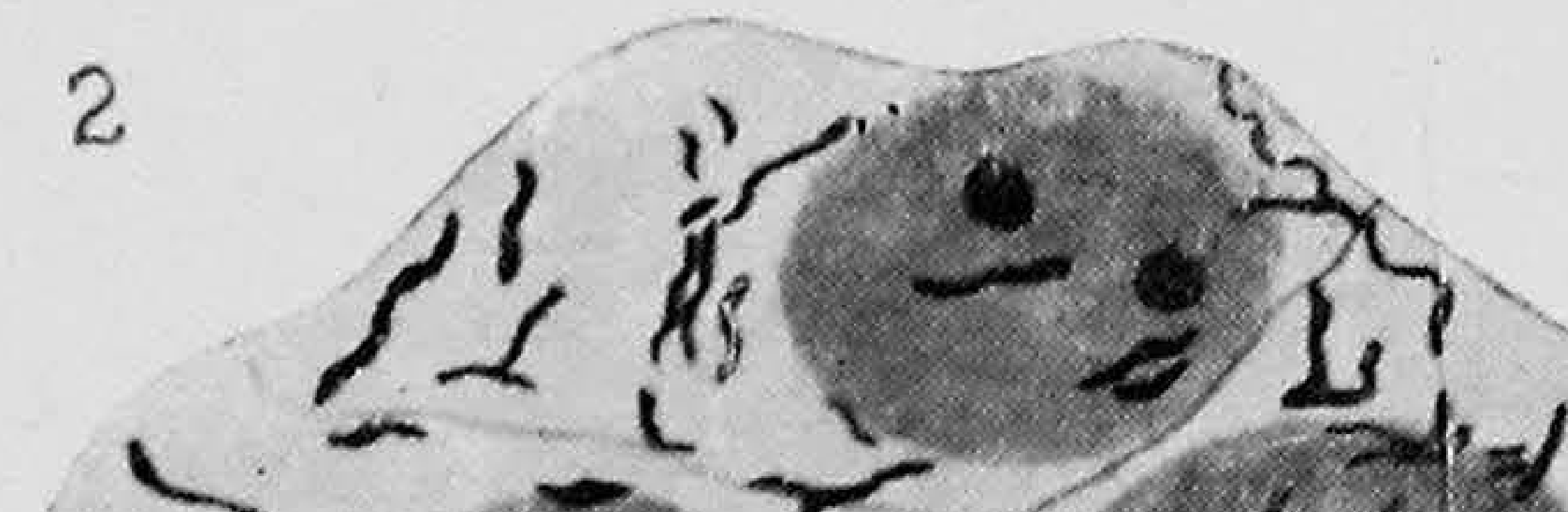

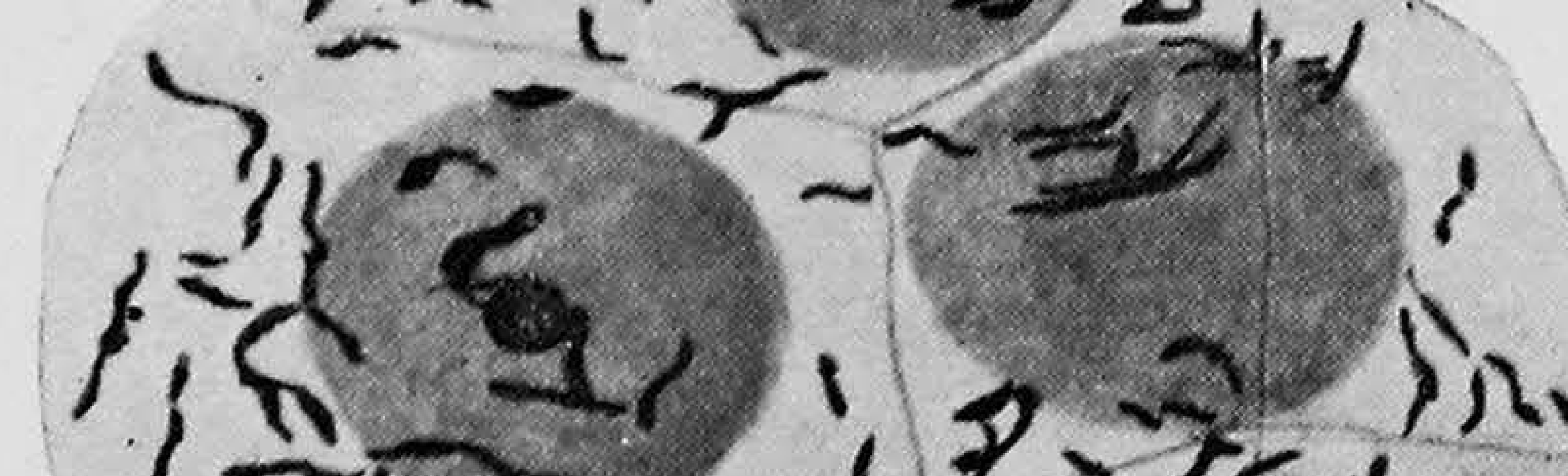

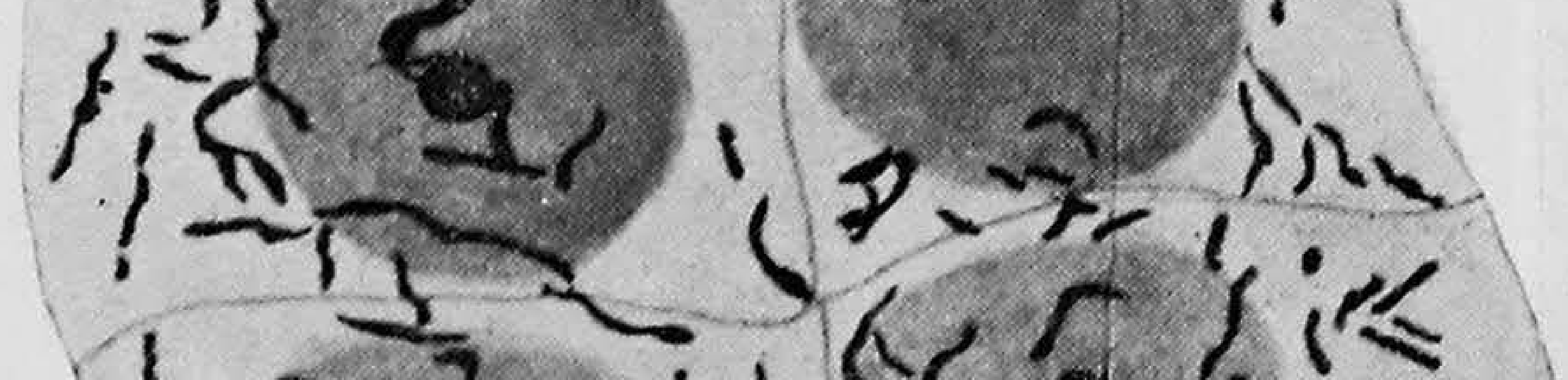
totive

$5 \quad 6.85 \times 24$

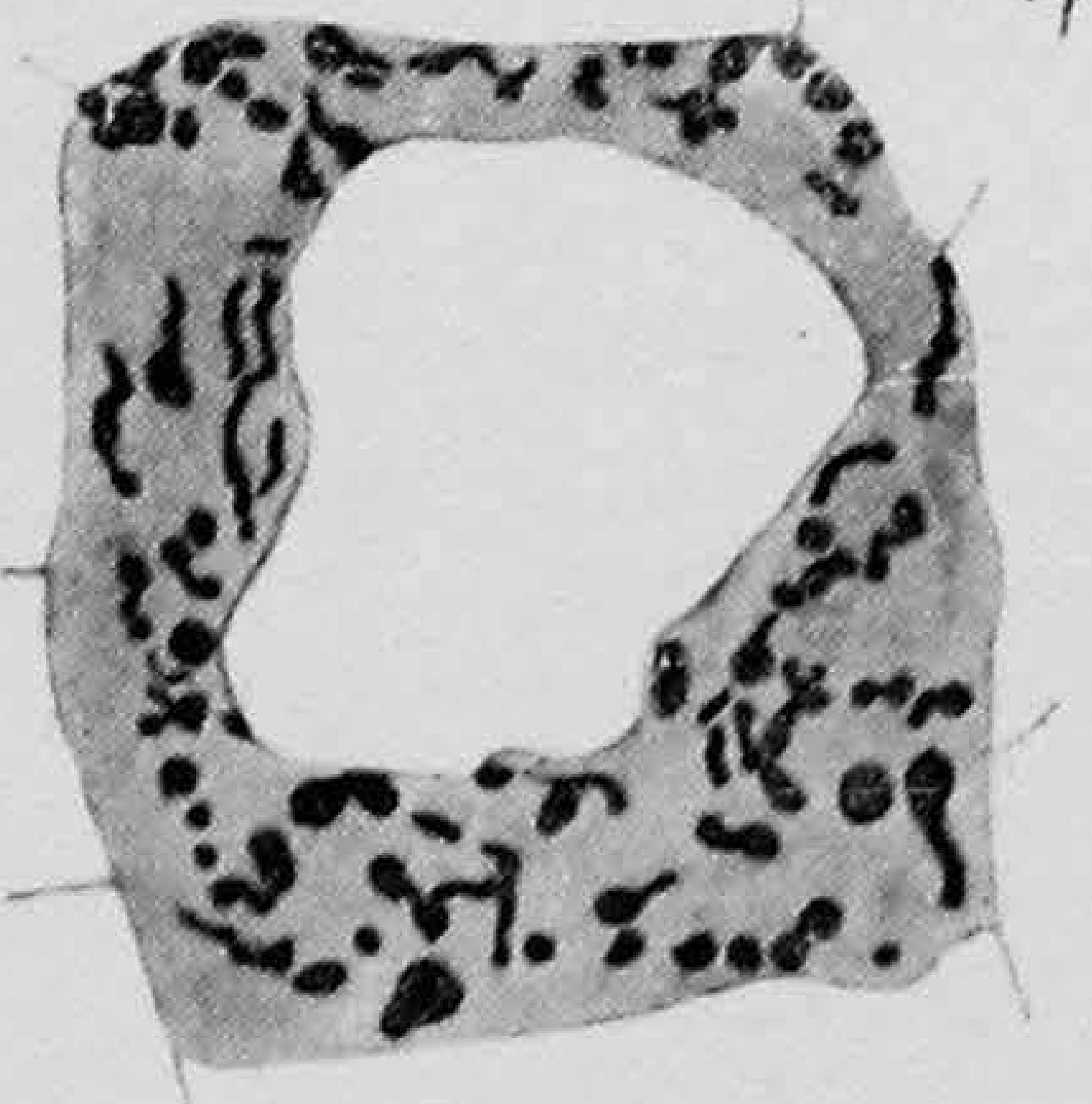

3

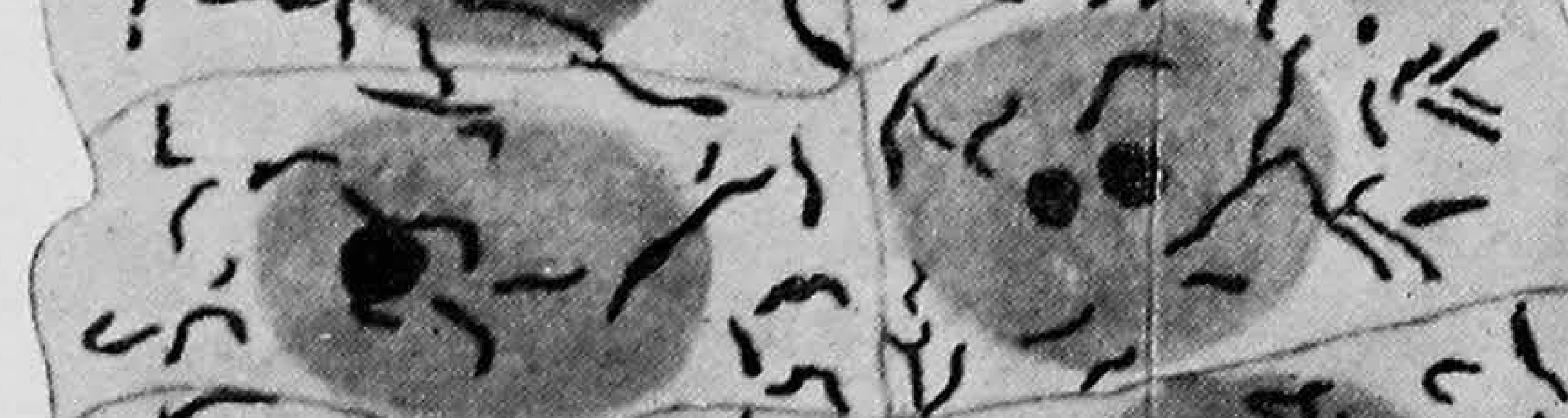

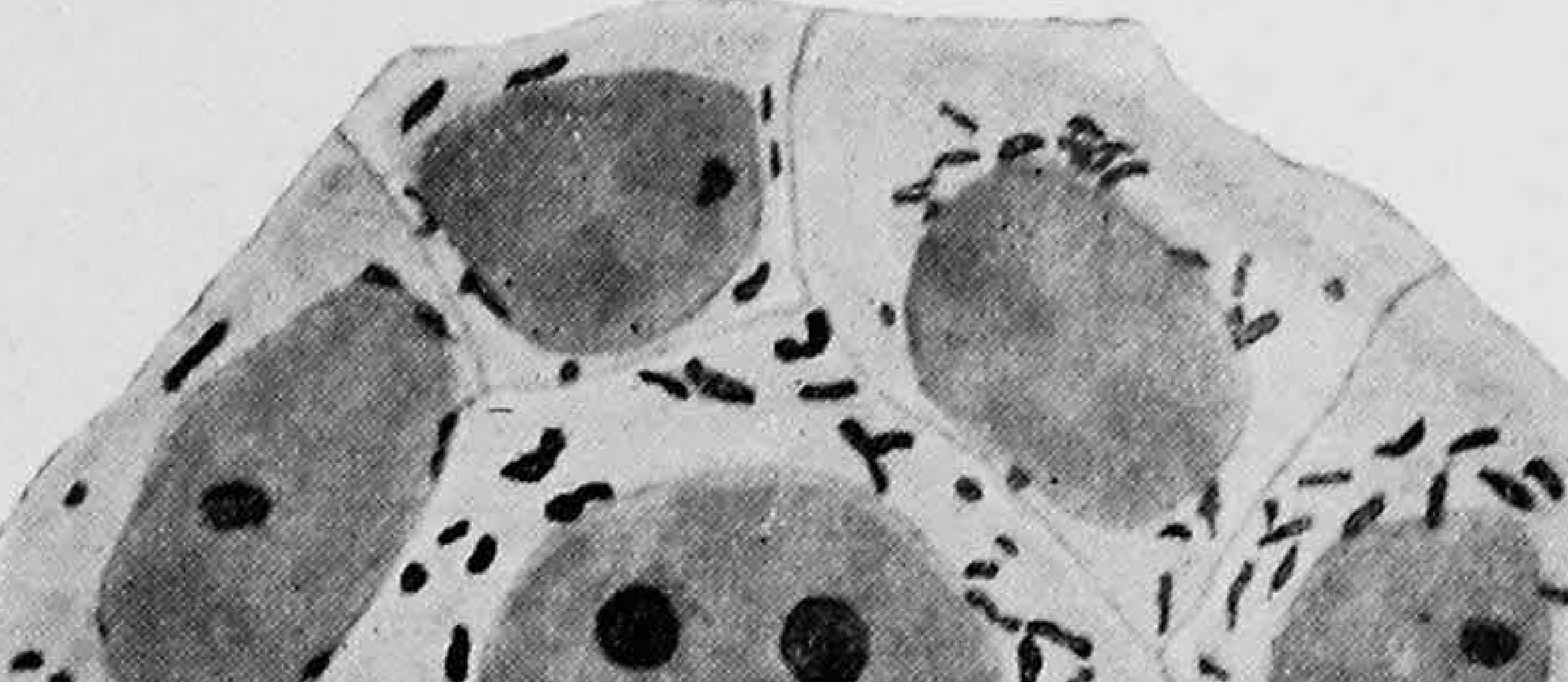

is i a o हो
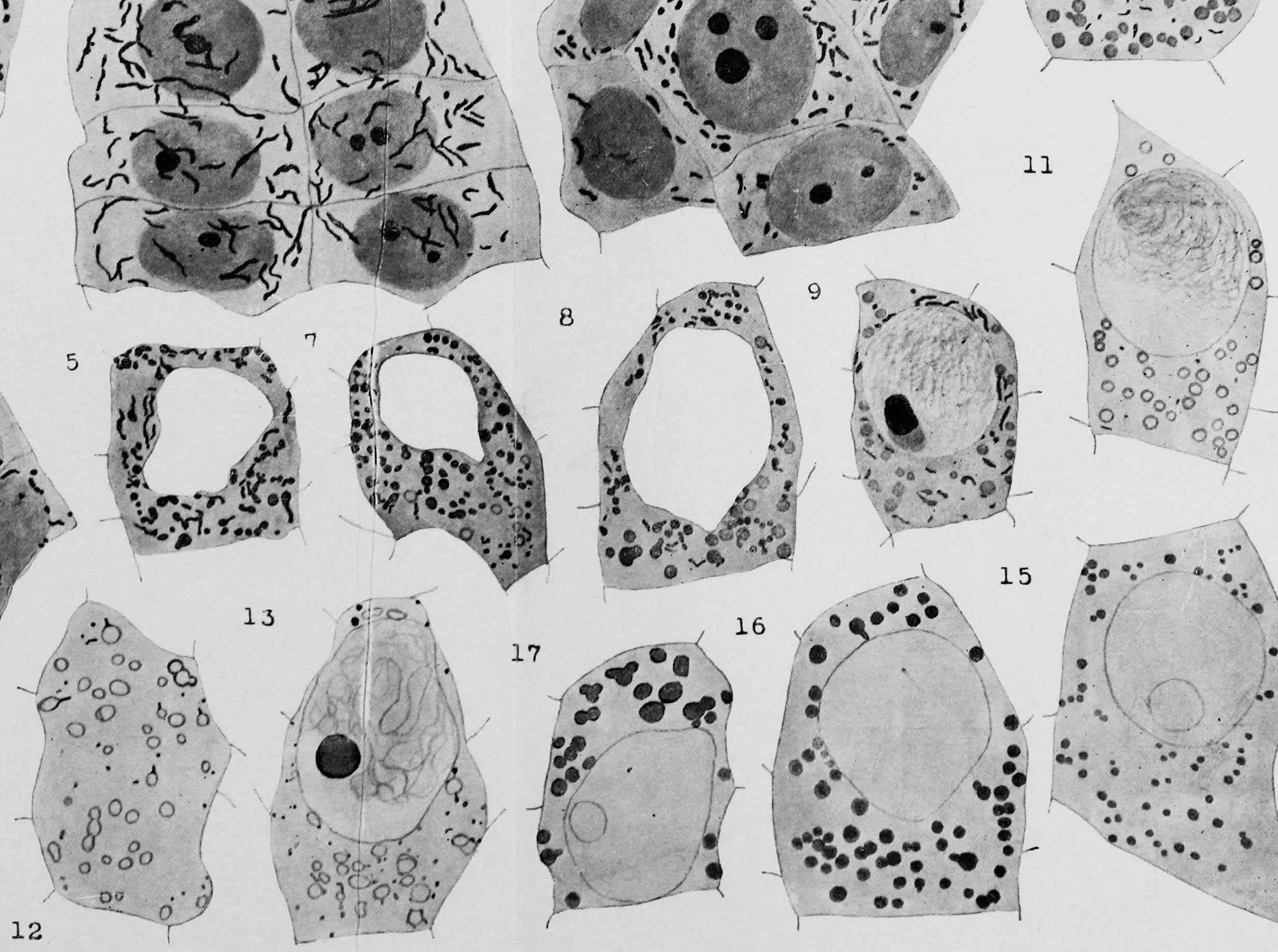

13

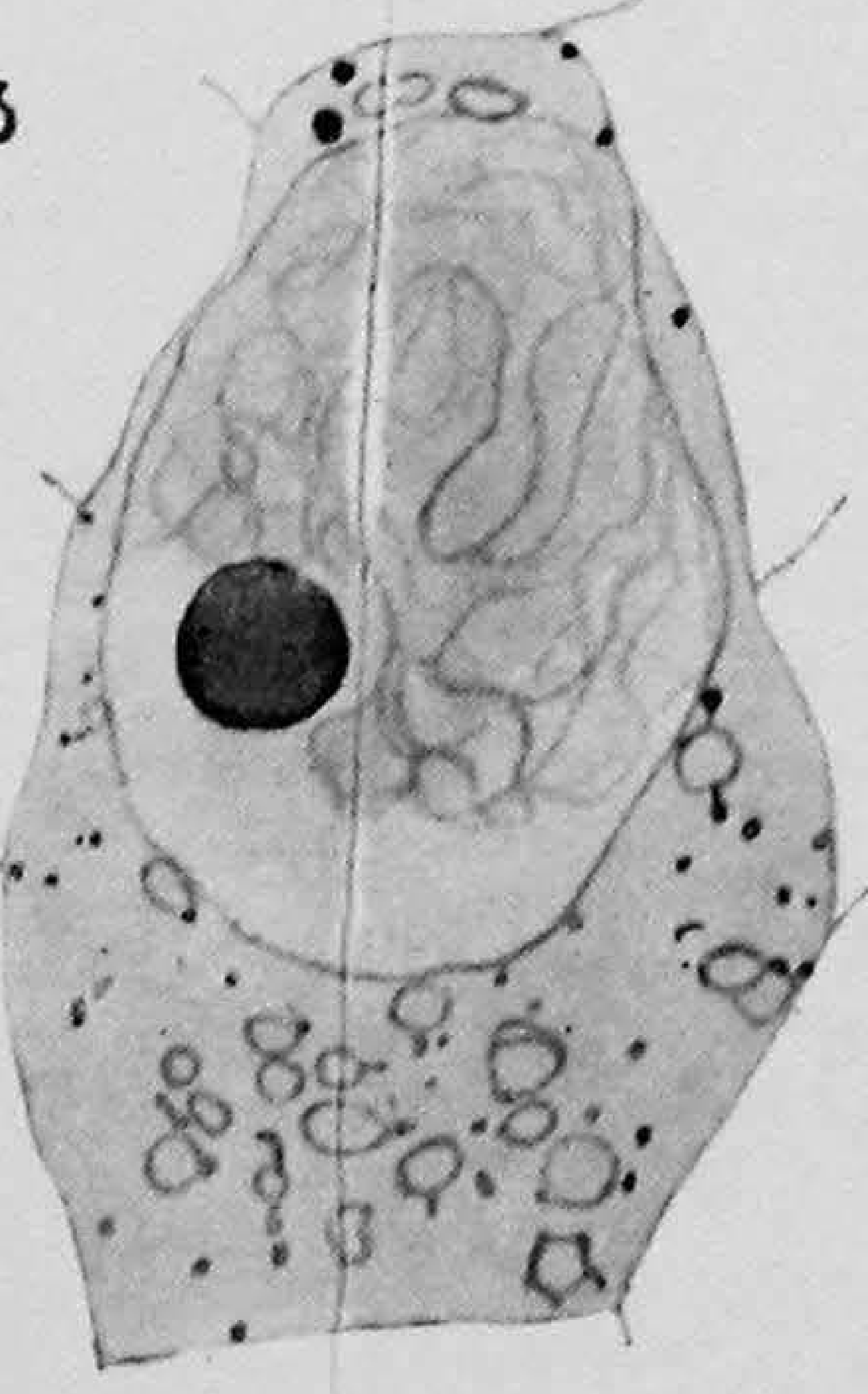

10

- 0.00 ó

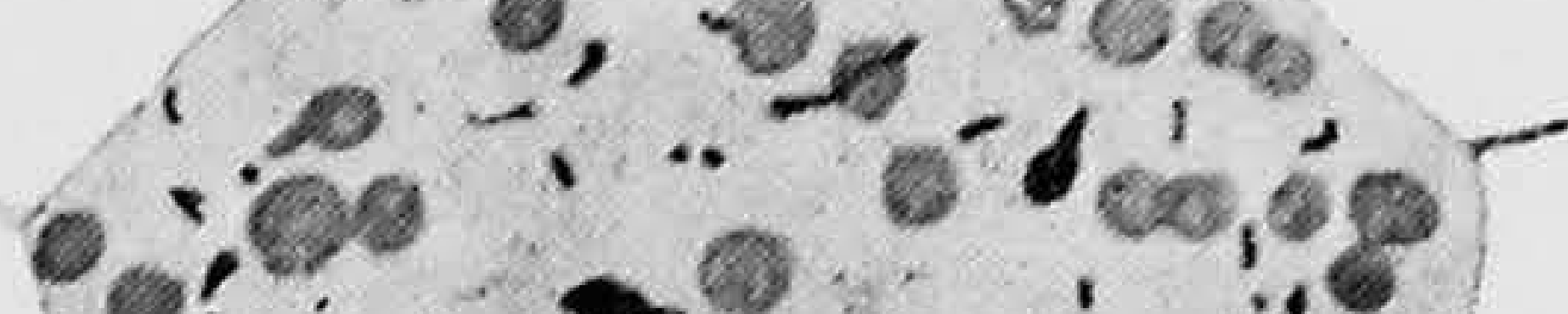
$1,1,2000$

II

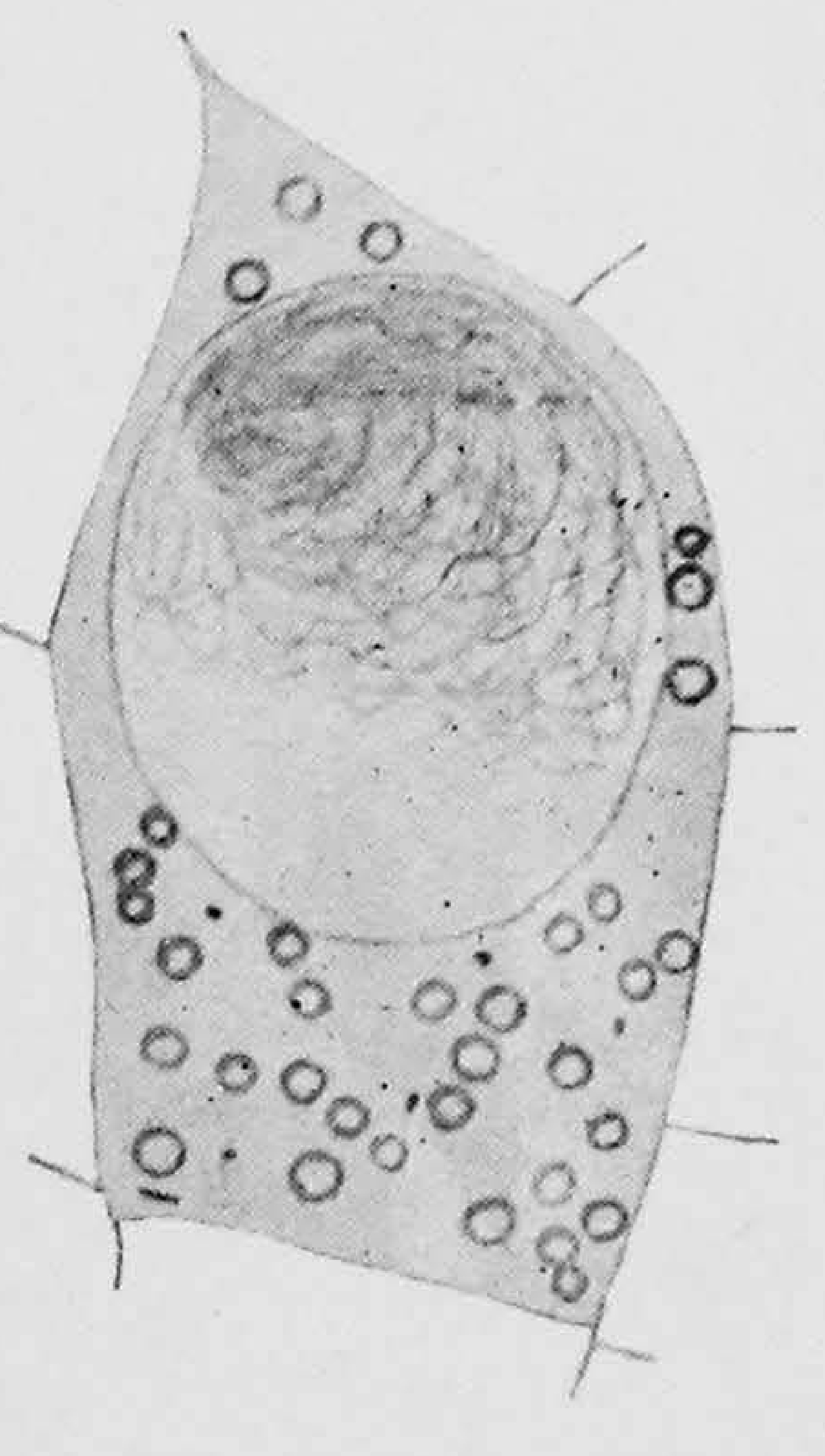
- $61000,-$
8

in
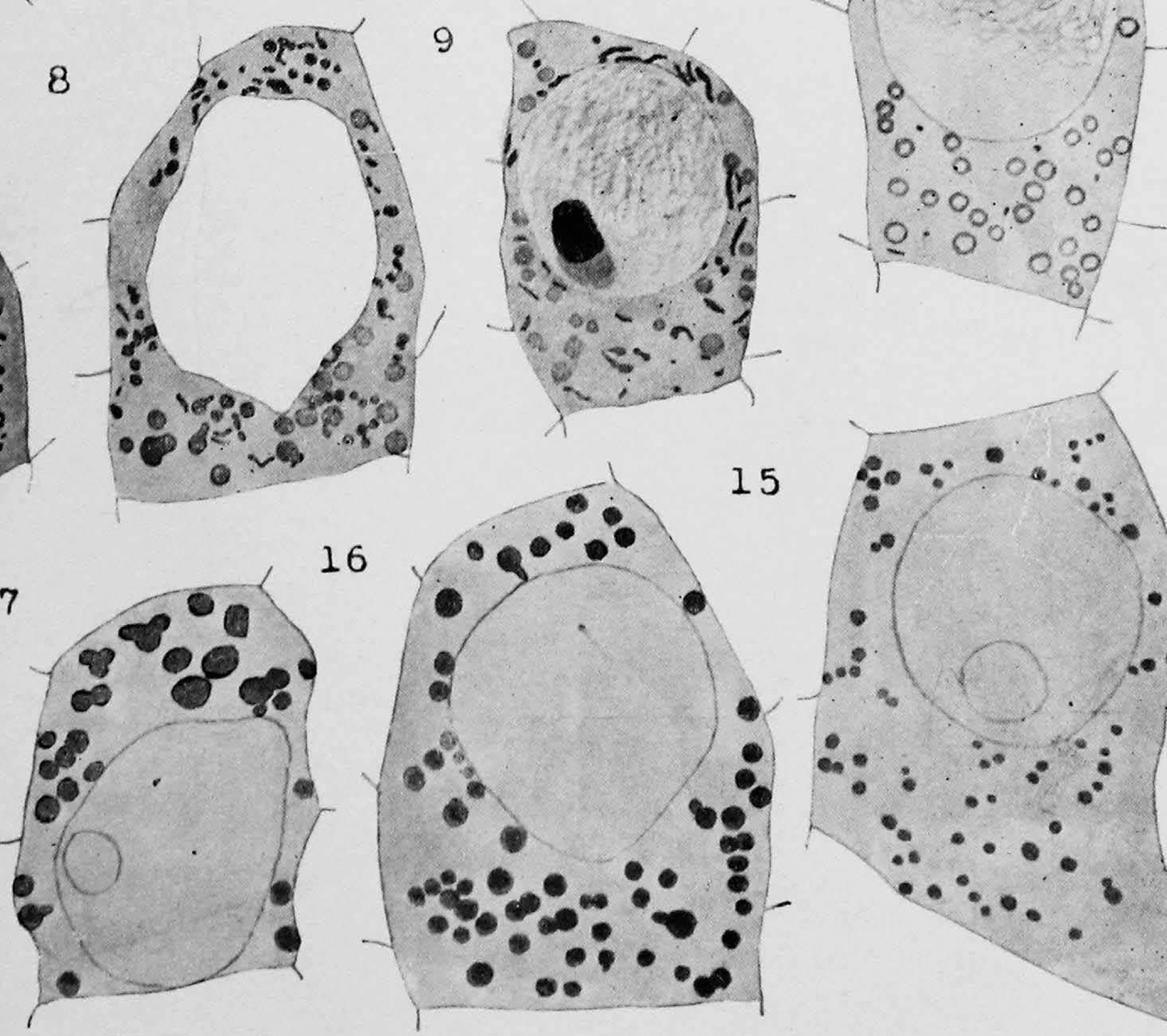

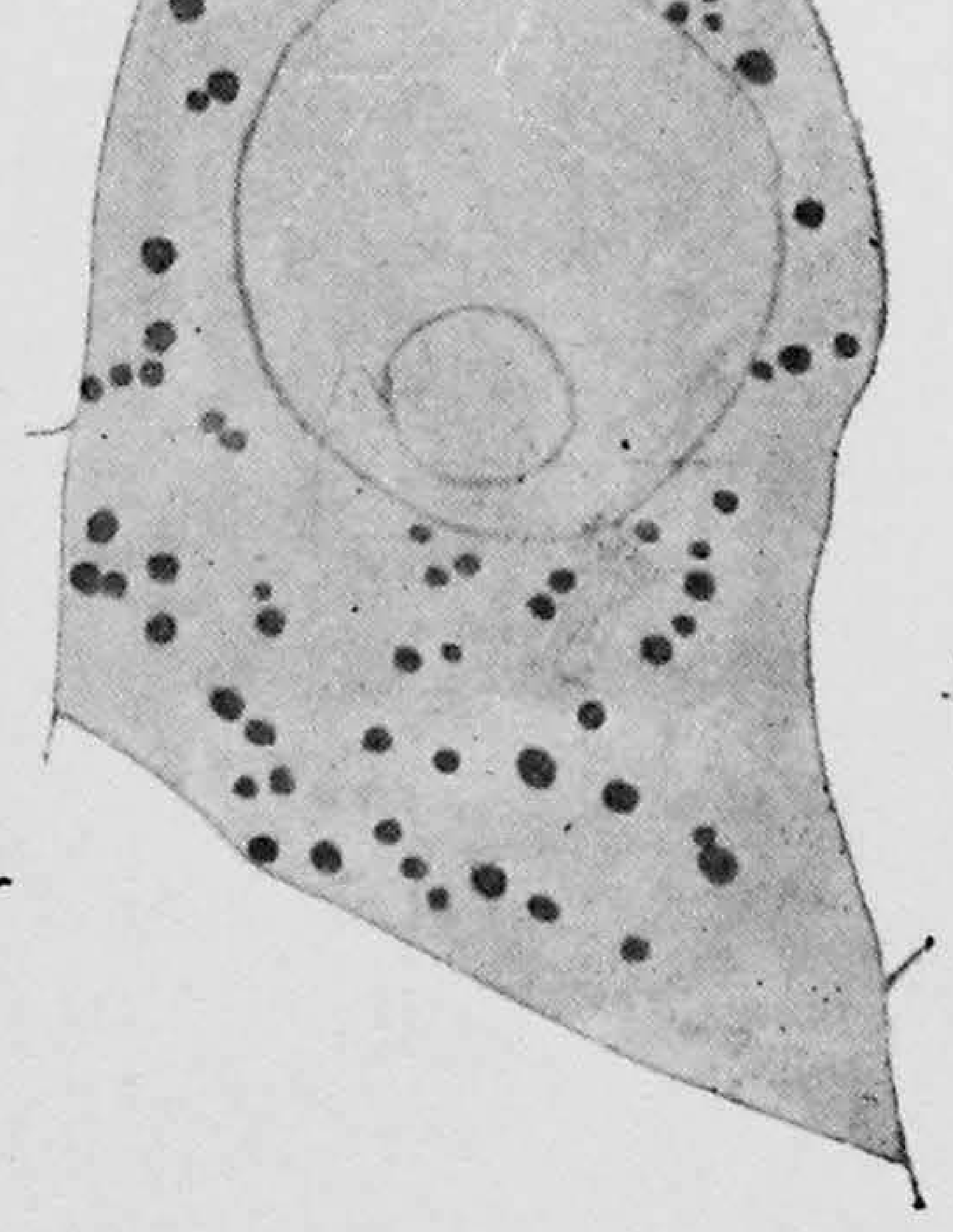



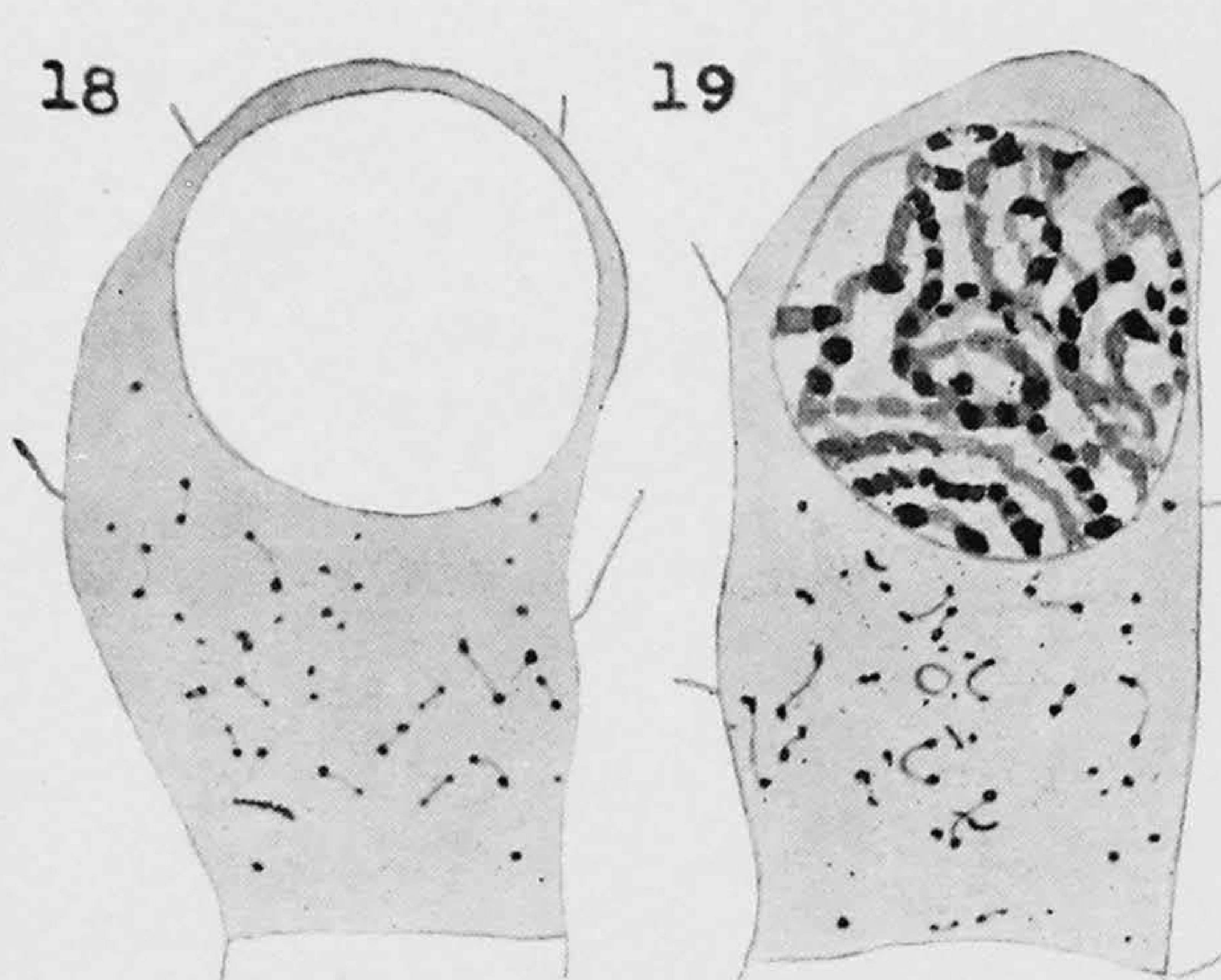

20

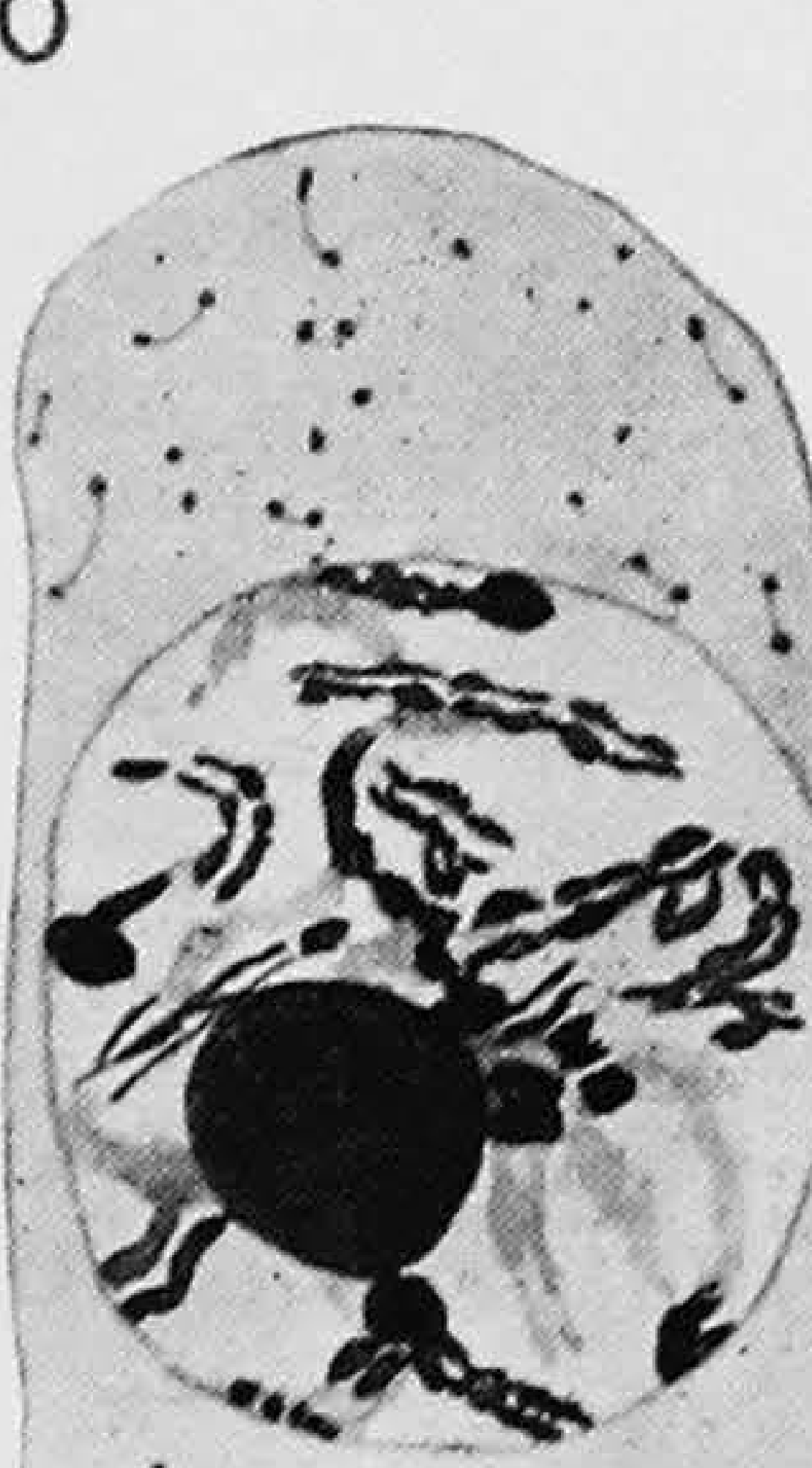

21

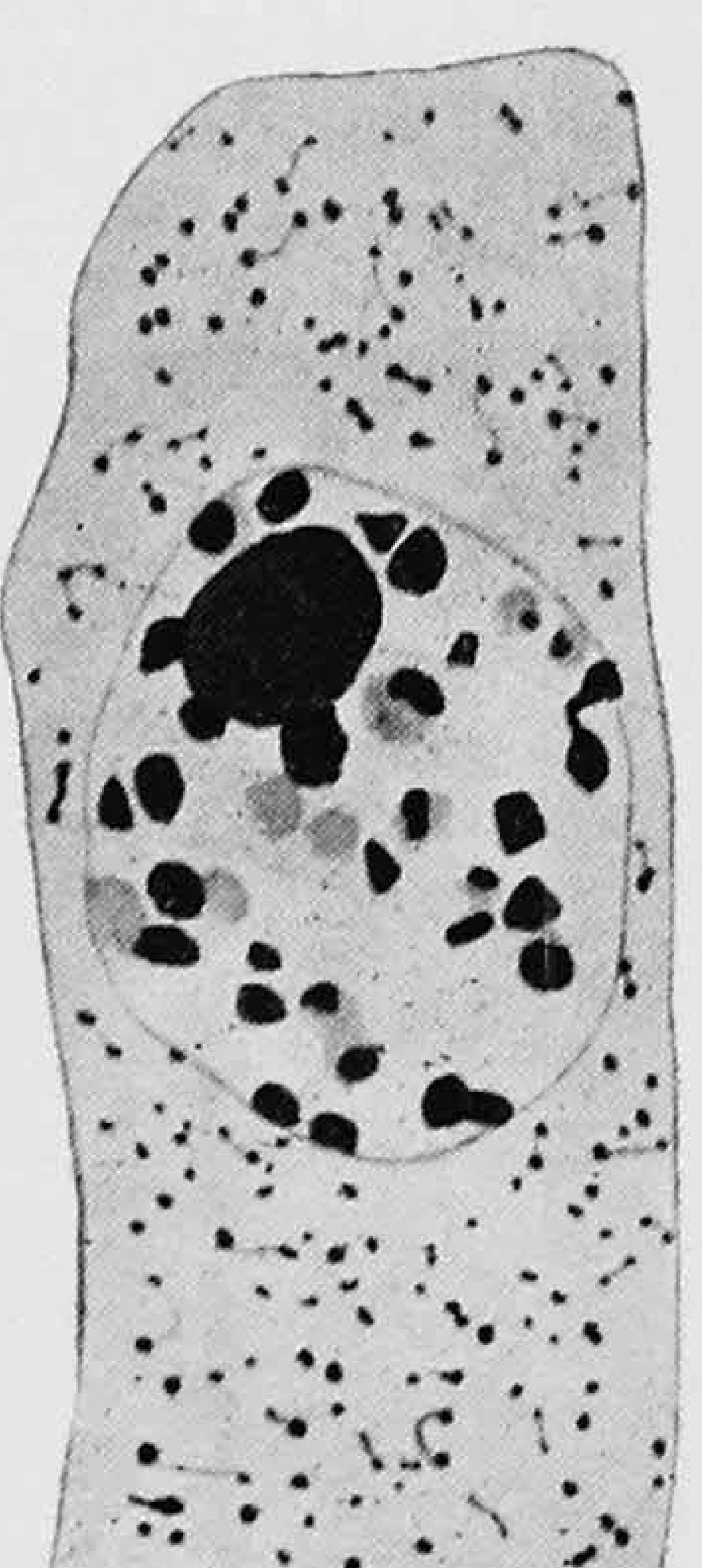

22
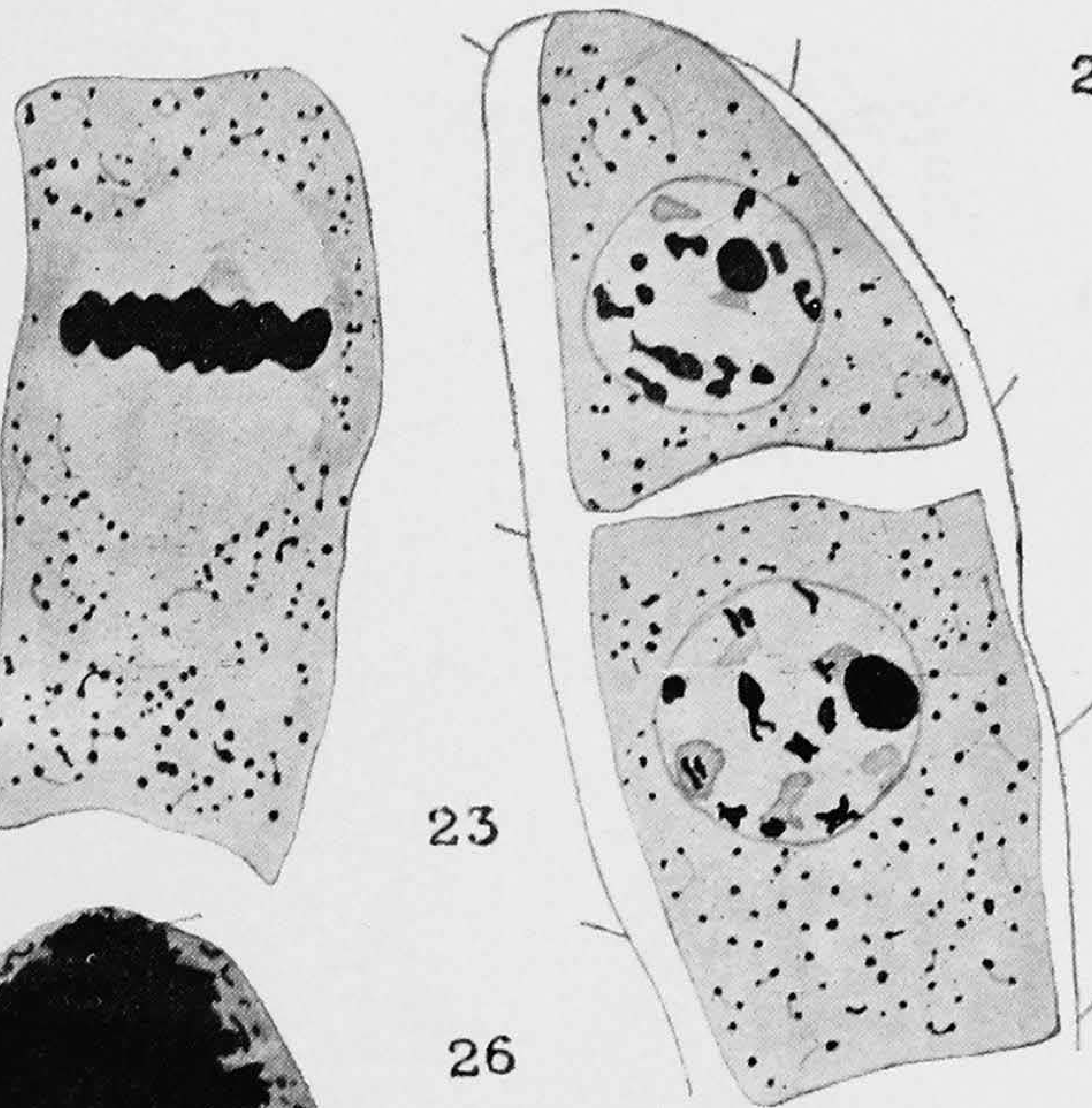

24
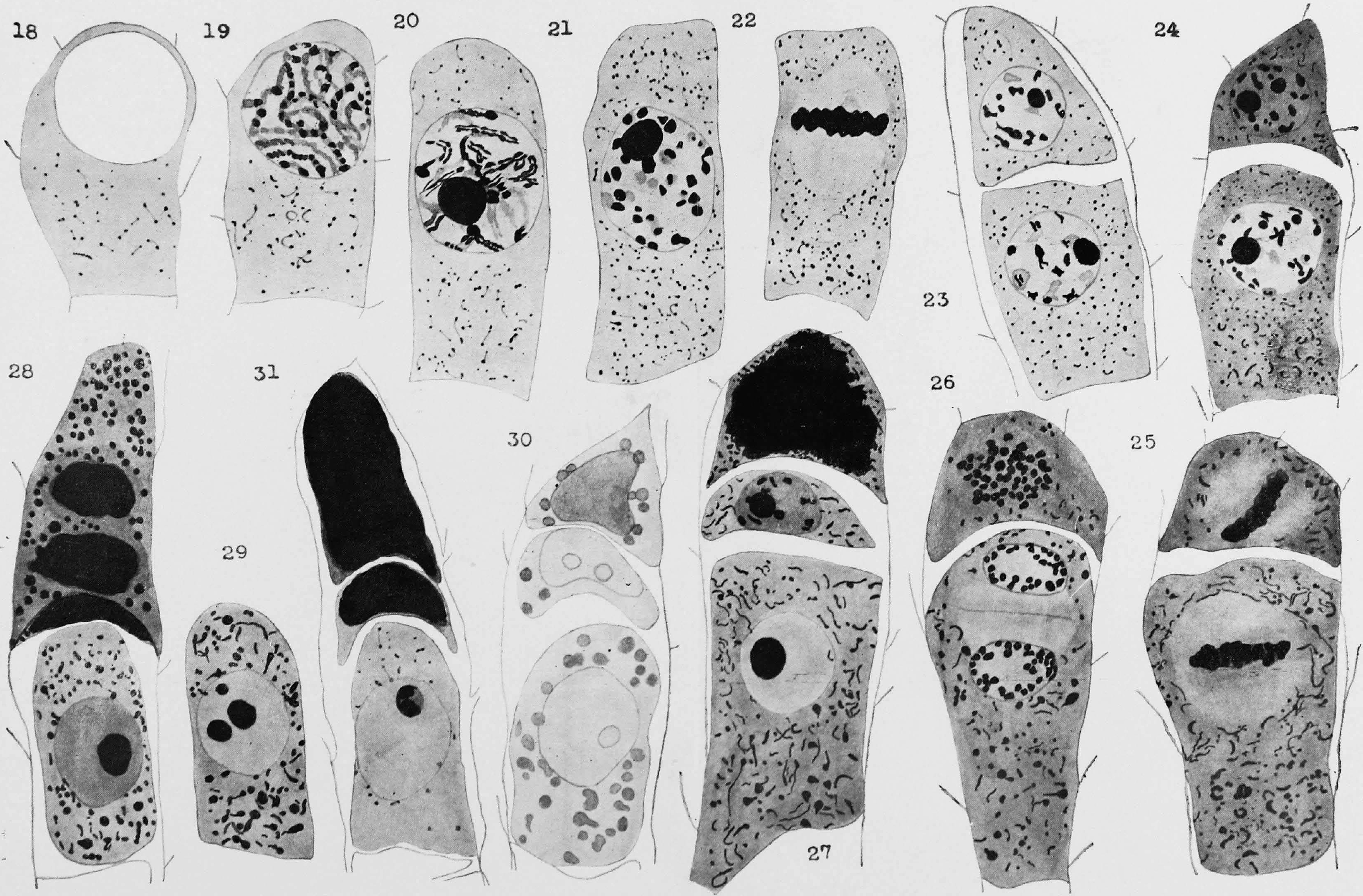


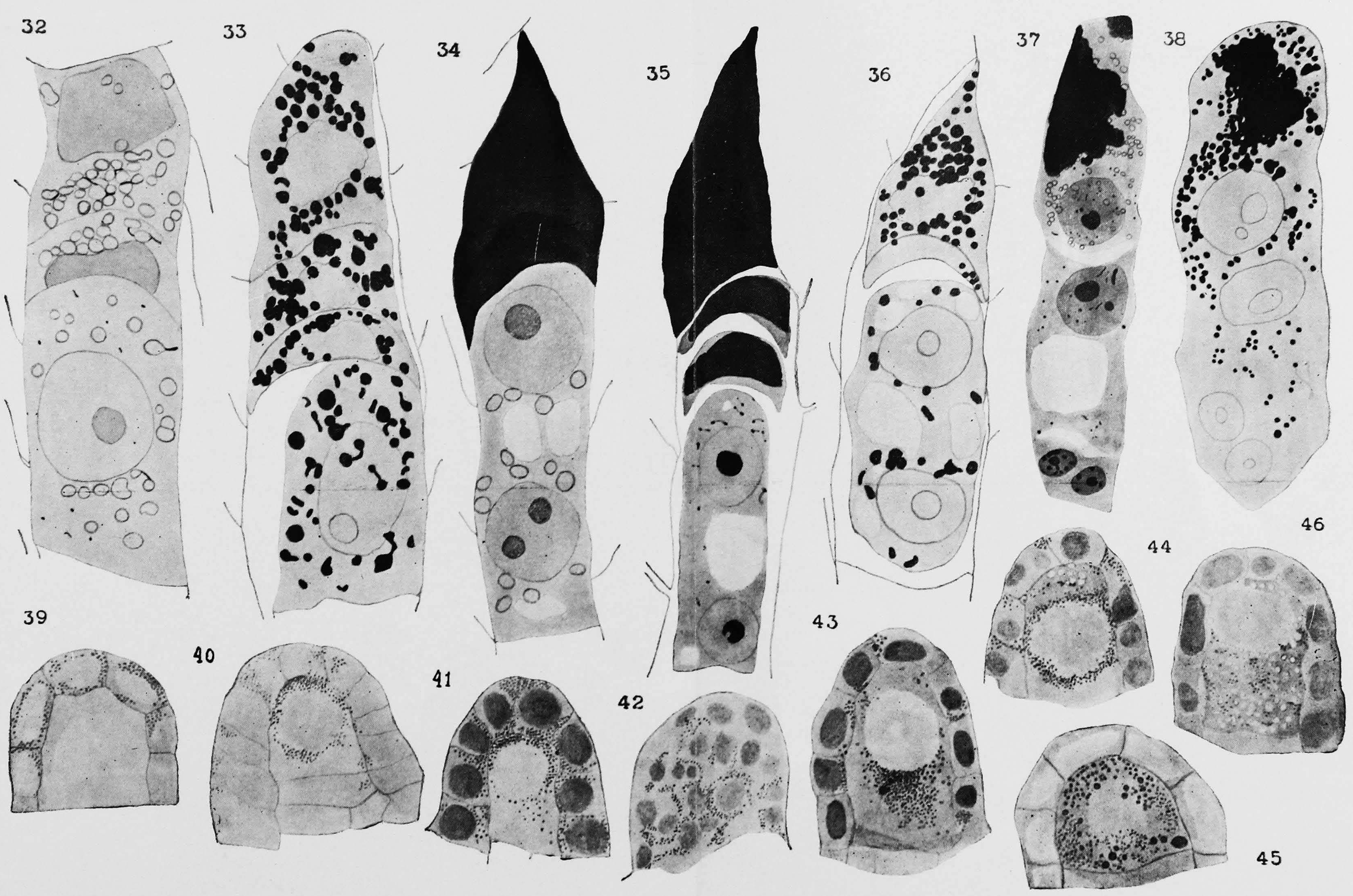

